\title{
COUPLED FLEXURAL - TORSIONAL VIBRATION AND STABILITY ANALYSIS OF PRE-LOADED BEAMS USING CONVENTIONAL AND DYNAMIC FINITE ELEMENT METHODS
}

\author{
by \\ Heenkenda Jayasinghe, B. Eng \\ Aeronautical Engineering
}

City University London, United Kingdom, 2011

\author{
A thesis presented to Ryerson University in partial \\ fulfillment of the requirements for the degree of \\ Master of Applied Science in the Program of \\ Aerospace Engineering
}

Toronto, Ontario, Canada, 2013

CHeenkenda Jayasinghe 2013 


\section{Author's Declaration}

\section{AUTHOR'S DECLARATION FOR ELECTRONIC SUBMISSION OF A THESIS}

I hereby declare that I am the sole author of this thesis or dissertation.

I authorize Ryerson University to lend this thesis or dissertation to other institutions or individuals for the purpose of scholarly research.

I further authorize Ryerson University to reproduce this thesis or dissertation by photocopying or by other means, in total or in part, at the request of other institutions or individuals for the purpose of scholarly research.

I understand that my thesis may be made electronically available to the public. 


\section{Abstract}

Dynamic Finite Element (DFE) and conventional finite element formulations are developed to study the flexural - torsional vibration and stability of an isotropic, homogeneous and linearly elastic pre-loaded beam subjected to an axial load and end-moment. Various classical boundary conditions are considered. Elementary Euler - Bernoulli bending and St. Venant torsion beam theories were used as a starting point to develop the governing equations and the finite element solutions. The nonlinear Eigenvalue problem resulted from the DFE method was solved using a program code written in MATLAB and the natural frequencies and mode shapes of the system were determined from the Eigenvalues and Eigenvectors, respectively. Similarly, a linear Eigenvalue problem was formulated and solved using a MATLAB code for the conventional FEM method. The conventional FEM results were validated against those available in the literature and ANSYS simulations and the DFE results were compared with the FEM results. The results confirmed that tensile forces increased the natural frequencies, which indicates beam stiffening. On the contrary, compressive forces reduced the natural frequencies, suggesting a reduction in beam stiffness. Similarly, when an end-moment was applied the stiffness of the beam and the natural frequencies diminished. More importantly, when a force and an end-moment were acting in combination, the results depended on the direction and magnitude of the axial force. Nevertheless, the stiffness of the beam is more sensitive to the changes in the magnitude and direction of the axial force compared to the moment. A buckling analysis of the beam was also carried out to determine the critical buckling end-moment and axial compressive force. 


\section{Acknowledgement}

I would like express my sincere gratitude to Prof. Seyed M. Hashemi for his continuous patience, wisdom, and guidance throughout my research, as well as for his motivation and enthusiasm when I needed them.

I would also like to thank my family and friends for all of their unwavering support along this journey. 


\section{Contents}

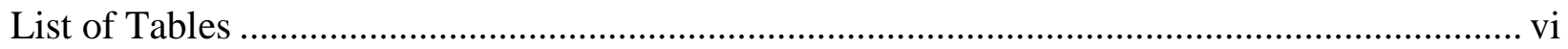

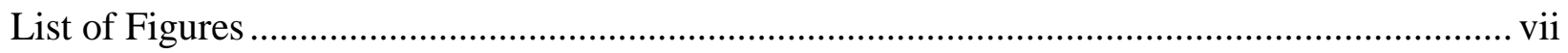

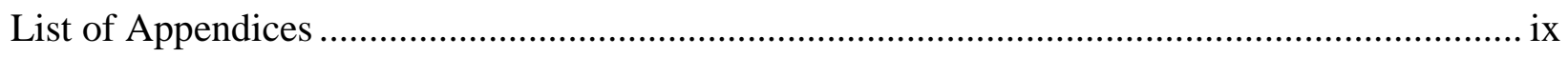

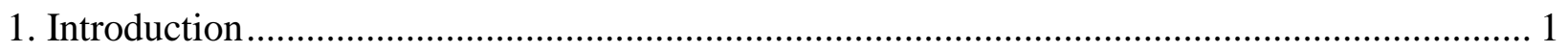

2. Conventional Finite Element Analysis .................................................................. 9

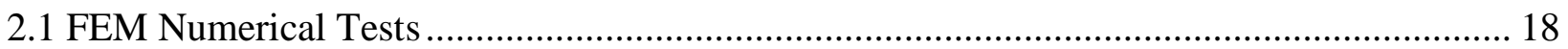

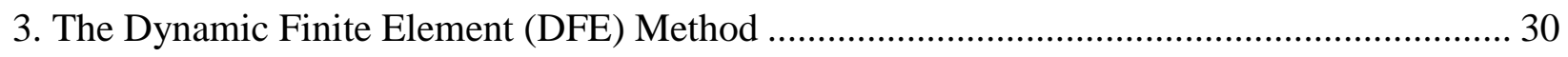

3.1 The Dynamic Stiffness Matrix method......................................................................... 30

3.2 The conventional FEM method based on polynomial interpolation functions..................... 30

3.3 The frequency- dependent Dynamic Finite Element method based on trigonometric shape

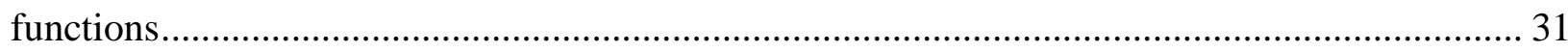

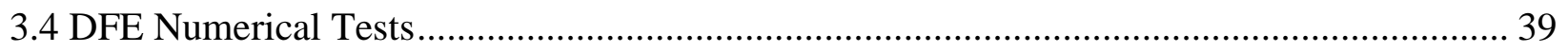

4. Concluding Remarks and Recommendations for Future Works ...................................... 51

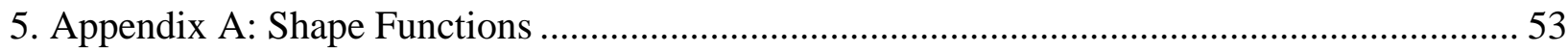

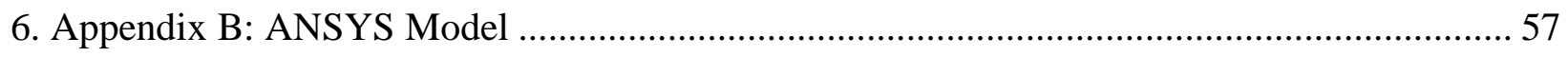




\section{List of Tables}

Table 1: Comparison between the exact and FEM results for the first three natural frequencies at $\mathrm{P}=0$ and $\mathrm{M}=0$ 18

Table 2: Convergence of the fifth natural frequency . 19

Table 3: First natural frequencies for cantilever boundary condition $(C-F)$ when force and moment are applied; a 40-element FEM model is used

Table 4: Fundamental frequencies for clamped - clamped boundary condition $(\mathrm{C}-\mathrm{C})$, when force and end-moment are applied; a 40-element FEM model is used 22

Table 5: First natural frequencies for pinned - pinned boundary condition $(\mathrm{P}-\mathrm{P})$ when force and moment are applied; a 40-element FEM model is used

Table 6: First natural frequencies for pinned - clamped boundary condition $(\mathrm{P}-\mathrm{C})$ when force and moment are applied; a 40-element FEM model is used 23

Table 7: Critical buckling moment for cantilevered boundary condition $(\mathrm{C}-\mathrm{F})$ with varying compressive force 23

Table 8: Critical buckling compressive force for cantilevered boundary condition $(\mathrm{C}-\mathrm{F})$ with varying end-moment.

Table 9: First natural frequencies for cantilever beam $(\mathrm{C}-\mathrm{F})$ when force and moment are applied 41

Table 10: First natural frequencies for clamped - clamped boundary condition $(\mathrm{C}-\mathrm{C})$ when force and moment are applied...... 42

Table 11: First natural frequencies for pinned - pinned boundary condition $(\mathrm{P}-\mathrm{P})$ when force and moment are applied 42 
Table 12: First natural frequencies for pinned - clamped boundary condition $(\mathrm{P}-\mathrm{C})$ when force and moment are applied. 43

Table 13: Critical buckling moment for cantilevered boundary condition $(\mathrm{C}-\mathrm{F})$ with varying compressive force 43

Table 14: Critical buckling compressive force for cantilevered boundary condition $(\mathrm{C}-\mathrm{F})$ with varying end-moment 44

\section{List of Figures}

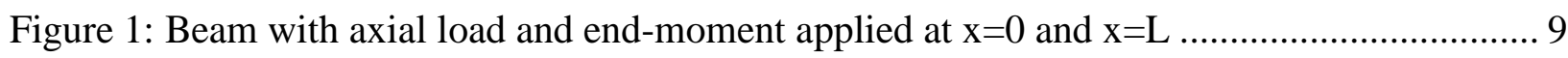

Figure 2: System discretized using elements with 3 degrees of freedom per node .................... 12

Figure 3: Convergence analysis for conventional FEM for cantilevered beam........................ 20

Figure 4:Variation of natural frequencies when tensile force and end-moment is applied for

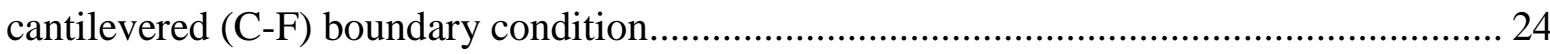

Figure 5: Variation of natural frequencies when tensile force and end-moment is applied for

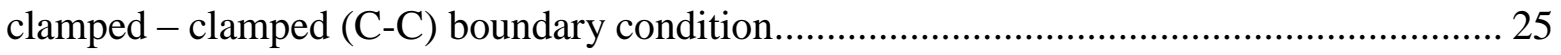

Figure 6: Variation of natural frequencies when tensile force and end-moment is applied for pinned - pinned (P-P) boundary condition 25

Figure 7: Variation of natural frequencies when tensile force and end-moment is applied for clamped - pinned (C-P) boundary condition. 26

Figure 8: Variation of critical buckling compressive force with end-moment .......................... 26

Figure 9: Variation of critical buckling end-moment with tensile and compressive force.......... 27

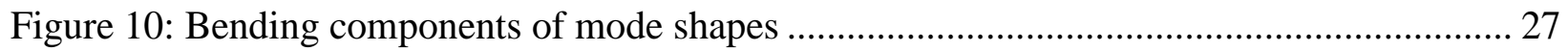

Figure 11: Torsional components of mode shapes ....................................................... 28

Figure 12: Convergence analysis for DFE method for cantilevered beam ............................. 40 
Figure 13: Comparison of convergence efficiency between DFE method and conventional FEM for cantilevered beam 41

Figure 14: Variation of natural frequencies when tensile force and end-moment is applied for cantilevered $(\mathrm{C}-\mathrm{F})$ boundary condition 44

Figure 15: Variation of natural frequencies when tensile force and end-moment is applied for clamped - clamped (C-C) boundary condition. 45

Figure 16: Variation of natural frequencies when tensile force and end-moment is applied for pinned - pinned (P-P) boundary condition. 45

Figure 17: Variation of natural frequencies when tensile force and end-moment is applied for pinned - clamped (P-C) boundary condition. 46

Figure 18: Variation of critical buckling end-moment with axial force for cantilevered (C-F) boundary condition 46

Figure 19: Variation of critical buckling compressive force with end-moment for cantilevered (C-

F) boundary condition 47

Figure 20: Bending component of mode shapes of the system 47

Figure 21: Torsional component of mode shapes of the system..... 48

Figure 22: Cubic Hermite shape functions for bending and linear shape functions for torsion used in conventional FEM 53

Figure 23: Frequency dependent trigonometric bending and torsion shape functions presented in [1] and [23] used in the DFE method 53

Figure 24: Dependency on the frequency of the first bending shape function $[1,23]$ used in the DFE method. 54 
Figure 25: Dependency on the frequency of the second bending shape function $[1,23]$ used in the DFE method.. 54

Figure 26: Dependency on the frequency of the third bending shape function $[1,23]$ used in the DFE method. 55

Figure 27: Dependency on the frequency of the fourth bending shape function $[1,23]$ used in the DFE method..... 55

Figure 28: Dependency on the frequency of the first torsional shape function $[1,23]$ used in the DFE method..... 56

Figure 29: Dependency on the frequency of the second torsional shape function $[1,23]$ used in the DFE method 56

Figure 30: Beam meshed in ANSYS using SOLID 187 elements........................................ 57

Figure 31: First coupled bending - torsion mode shape from ANSYS ................................ 57

Figure 32: Second coupled bending - torsion mode shape from ANSYS ..............................58

Figure 33: Third coupled bending - torsion mode shape from ANSYS ................................ 58

Figure 34: Fourth coupled bending - torsion mode shape from ANSYS .............................. 59

Figure 35: Fifth coupled bending - torsion mode shape from ANSYS .................................59

\section{List of Appendices}

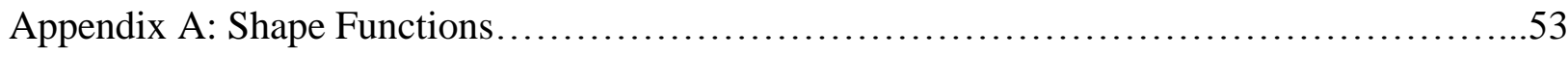

Appendix B: ANSYS Model..................................................57 


\section{Introduction}

Beams are important and commonly used structures, since many components of airborne vehicles, such as wings and helicopter blades could be modeled as a simple beam or as a series of beams during the preliminary design stages. Additionally, multiple layers of beams could be used to model sandwich/composite materials and delamination problems.

Such an aircraft structural component is exposed to a large range of vibrational frequencies during its operational lifetime. Therefore, it is of utmost importance to study the vibrational characteristics, such as the fundamental frequencies and mode shapes of a system in order to avoid resonance. Thus, prior to manufacturing, most components undergo modal analyses which would allow the designers to investigate the effects of various loading and boundary conditions the part would be subjected to during its operation, and understand the effect of those on the vibrational characteristics of the component. Using these results, the engineers and designers could then alter and optimize the geometry of the system and the materials used, to gain a favorable outcome. They would also be able to determine the most suitable locations to add supports and areas that require additional reinforcements. Thus, modal analysis would ensure that the natural frequencies of the component is maintained within an accepted range.

In many aerospace applications, the structural beam elements are in a state of preload or pre stress due to the application of axial loads and end-moments (e.g. helicopter rotor blades and structural elements attached through semi - rigid connections). The centrifugal force acting on the blades could be modelled as an axial force. The presence of such axial loads and end-moments can affect the vibrational and stability characteristics of the beam and as such it is worthwhile to investigate. Therefore, in this study a modal analysis will be carried out to investigate the stability 
and vibration of a simple Euler - Bernoulli beam under various boundary conditions subjected to axial force and end- moments.

Many researchers have studied the free vibration of isotropic, sandwiched, composite and thinwalled beams subjected to various loading configurations, using numerous techniques. Hashemi and Richard [1] developed a new Dynamic Finite Element (DFE) formulation to analyse the free vibration of bending - torsion coupled beams. The flexural axis of the beam studied by Hashemi et al. is not coincident with the inertial axis. Hashemi and Roach [2] also formulated a DFE for the free vibration of an extension - torsion coupled composite beam. A quasi - exact DFE formulation, for the free vibration analysis of a three layered sandwich beam consisting of a thick, soft, low strength and density core as well as two face layers made of high strength material, was developed by Hashemi and Adique [3]. Borneman and Hashemi [4] developed a DFE for the free vibration analysis of a bending - torsion coupled laminated composite wing beam. The effects of shear deformation and rotary inertia were neglected in this study, but were accounted for in another study by Hashemi and Pereira [41].

The exact method has been used to determine the flexural - torsional vibrational characteristics of a uniform beam with single cross sectional symmetry by Dokumaci et al [5]. The classical finite element method was used by Mei [6] to study the coupled vibration of thin walled beams with open section. This study included the effects of warping stiffness. The free vibration of an open, variable cross section I-beam was investigated by Wekezer [7]. The flexural - torsional vibration of a uniform beam was studied by Tanaka et al, [8], by determining the exact solution of the governing differential equations.

Bannerjee et al. have used the Dynamic Stiffness Matrix Method (DSM), which utilizes the general closed form solution of the governing differential equations of motion to generate the frequency 
dependent stiffness matrix, for vibrational analysis of isotropic $[9,10]$, sandwich $[11,12,13]$ and composite [14] beams in the past. Banerjee et al. [15] also developed an exact DSM to investigate the vibration of an Euler - Bernoulli thin walled beam and exploited the Wittrick - Williams [16] root finding algorithm to arrive at the Eigensolutions. Bannerjee and Su [17] later used the DSM to conduct a free, transverse and lateral vibration analysis of a beam coupled with torsion. Borneman et al. [18] also used the DSM method to investigate the vibrational characteristics of a doubly coupled (material and geometric) composite beam. Friberg [19] and Leung [20] developed an exact DSM of a thin walled beam. The Dynamic Stiffness method was used by Hallauer et al. [21] to determine the vibrational characteristics and generalized masses of an aircraft wing modelled as a series of three simple beams.

The axial load and end-moment affect the stability and vibrational characteristics of the beam. The effect of the axial load on the transverse vibrational characteristics of beams has been well established [22]. Hashemi and Richard [23] conducted a vibrational analysis on an axially loaded bending - torsion geometrically coupled beam using the DFE method. Banerjee et al. [24] studied the coupled flexural - torsional vibration of an axially loaded Timoshenko beam. Analytical solutions were developed by Bannerjee et al. [25, 26] to model a uniform, axially loaded, cantilevered beam with flexural - torsional geometric coupling as a result of non-coincident shear and mass centers. The effects of warping has been neglected in these studies. Jun et al. [27] studied the coupled flexural - torsional vibration of an axially loaded, thin walled beam with monosymmetrical cross sections and accounted for the effects of warping. The effect of axial load has also been previously studied by Murthy and Neogy [28] for clamped and pinned boundary conditions as well as by Gallert and Gluck [29] for the cantilevered boundary condition. Bokaian [30] studied the natural frequencies of a uniform single span beam subjected to a constant tensile 
axial load for various boundary conditions. The same author also investigated the vibrational characteristics of a uniform single span beam for ten different end conditions when a constant compressive axial load is applied [31]. Shaker et al. [32] conducted a modal analysis to determine the effect of axial load on the mode shapes and natural frequencies of beams.

It has also been found by Chen and Astuta [33] that transverse bending and torsion is coupled by static end-moments and that flexural - torsional buckling is comprised of this transverse flexure and torsion. Analytical investigations on the influence of axial loads and end-moments on the vibration of beams have been previously reported by Joshi and Suryanarayan for a simply supported case [34] as well as for various boundary conditions [35]. Joshi and Suryanarayan [36] also studied analytically, the flexural - torsional instability of thin walled beams subjected to axial loads and end-moments. The same authors studied the coupled bending - torsion vibration of a deep rectangular beam that was initially stressed due to the application of moments varying along the span of the beam [37]. Pavlovic and Kosic [38] developed a closed form analytical solution to investigate the effects of end-moments on a simply supported thin walled beam. Pavlovic et al. [39] also formulated the analytical solutions to study a simply supported thin walled beam subjected to the combined action of an axial force and end-moment.

The reliability and accuracy of such modal analysis results depends on the method implemented. There are several analytical, semi-analytical and numerical methods that could be used to carry out the modal analysis. All methods mentioned in the above references have their inherent advantages and disadvantages. Although there exists a class of problems for which an exact solution can be easily obtained, in most cases an exact solution for the normal modes and frequencies of the system would be intractable. Exact methods such as DSM are capable of using just one element matrix to produce the exact results for the vibrational characteristics of a beam. 
Nevertheless, the applicability of such analytical methods is limited to simple and special cases. With every change made to the system configuration, the equations should be reformulated and it is difficult to use analytical methods to model problems with variations in geometry and material properties. Thus, recourse would be made to one of the many approximate solutions such as the Rayleigh-Ritz method and Galerkin's method [40].

The conventional Finite Element Method (FEM), which uses the Galerkin method of weighted residuals, is widely used for modal analysis and is very popular among researchers since it is convenient and adaptable to many complex systems including systems consisting of material and geometric variations. Geometric variations are modelled as stepped, piecewise - uniform configurations. In this method, the system is discretized once the weak form of the governing differential equations is obtained by applying the Galerkin method of weighted residuals. Cubic Hermite approximations are frequently used for transverse flexural displacements and linear approximations for torsion. This results in the element mass and stiffness matrices, which are independent of the natural frequency. Assembling the element mass and stiffness matrices creates a linear Eigenvalue problem of which, the Eigenvalues and Eigenvectors give the natural frequencies and mode shapes of the system, respectively.

The DFE method [1,23] is a hybrid frequency-dependent approximate solution method which is more accurate than the conventional FEM and, unlike the DSM, is adaptable to many complex configurations. It allows for a reduced mesh size and its formulation is quite similar to that of the conventional FEM. The Galerkin method of weighted residuals is also used in the DFE method to arrive at the weak integral form of the governing differential equations, after which the DFE formulation process deviates from the classical FEM. Instead of using polynomial and linear shape functions to approximate the flexural and torsional displacements, respectively, the DFE method 
utilizes frequency-dependent basis/shape functions presented in [1] and [23] to approximate the displacements. The trigonometric shape functions have been obtained in [1] and [23] by taking the exact solutions for the differential equations of motion governing the uncoupled vibrations of a uniform beam. The nodal approximations of displacement is found using the frequency-dependent shape functions obtained when the solutions to the above governing differential equations are employed as basis functions. Subsequently, discretization of the beam is carried out similar to the conventional FEM, which leads to the frequency-dependent dynamic element stiffness matrices. When these element matrices are assembled, the frequency-dependent global dynamic stiffness matrix is found. In order to find the natural frequencies of the system, the frequency, $\omega$, is swept to search for particular frequencies that would make the determinant of the global stiffness matrix go to zero. The corresponding eigenvector provides the mode shapes of the system.

To the best of the author's knowledge, a conventional or dynamic finite element formulation has not yet been developed to model the geometrically coupled flexural - torsional free vibration of an Euler - Bernoulli beam subjected to an axial force and an end-moment simultaneously. Therefore, in what follows, a classical finite element solution and a DFE formulation are presented to investigate the stability and flexural - torsional vibration of a simple Euler - Bernoulli beam subjected to an axial load and an end-moment. The effects of the axial load and end-moment on the stiffness and natural frequencies of the beam for various classical boundary conditions are examined. Program codes were written for the FEM and DFE methods using MATLAB and the results for the FEM code were validated using ANSYS commercial software. Subsequently, the DFE code results were compared for accuracy using the results produced for the same mathematical model, by the FEM code. 
The purpose of developing a DFE solution for a beam model that is axially loaded and geometrically coupled due to an end-moment thus inducing flexural - torsional vibration, is that it could be then used as a powerful tool to quickly investigate the vibrational and stability characteristics of numerous aerospace applications that are modelled as beams or assemblages of several identical beams (planar or space frames) to a very high degree of accuracy at the preliminary design stage. It is very important to take in to account the coupling effects in vibration and response calculations of these types of structures. The DFE beam model could be conveniently used to study periodical structures or more complex assemblies made of several identical substructures (beams) that has the same dynamic stiffness components and frequency characteristics.

Given the magnitude of aerospace components that are axially loaded and bending-torsion coupled that could be represented as beams to an acceptable degree of accuracy during the preliminary design stages, such as helicopter, propeller, compressor and turbine blades, the fact that engineers and designers could arrive at an acceptable ballpark for the vibrational characteristics within a fraction of the time, especially for higher modes, using an extremely coarser mesh in comparison to conventional FEM is a massive advantage as it avoids the difficulty of having to solve a very large Eigenvalue problem. For such aerospace components the coupled bending-torsional frequencies and mode shapes are crucial for aeroelastic calculations. The fact that any mode number could be investigated using dynamic elements regardless of the total number of degrees of freedom of the global system, is also an advantage.

Therefore, in the second chapter the problem that is studied will be explicitly defined and the theoretical aspects involved in developing the conventional FEM method will be explained in detail commencing from the governing differential equations of motion and extending to the point 
where the linear Eigenvalue problem is developed. The element mass and stiffness matrices resulting due to the discretization process will also be presented here.

Next, the numerical test results obtained from the conventional FEM analysis and a verification of the FEM results using ANSYS simulations would be presented. Comments will be made on the accuracy and efficiency of the solution.

In the third and penultimate chapter, the Dynamic Finite Element formulation process would be presented and its distinctions from the conventional FEM method would be elaborated. The process leading to the development of the coupled and uncoupled element stiffness matrices and the frequency dependent nonlinear Eigenvalue problem will be discussed in detail.

This will be followed by the results of the numerical tests performed using the DFE method which will be compared for accuracy and efficiency of convergence with the conventional FEM results.

Finally, conclusions will be drawn upon the results presented previously and the benefits and applications of the developed DFE beam model would be discussed. Additionally, this chapter will also be comprised of comments on the extendibility of the current work to incorporate other effects that were not considered in this study such as warping, geometric nonlinearity and variation of material properties that could be useful for future works. 


\section{Conventional Finite Element Analysis}

Consider a linearly elastic, homogeneous, isotropic beam subjected to an end-moment, $M$, and an axial load, $P$, undergoing linear vibrations. Euler - Bernoulli bending and St. Venant torsion beam theories are used to derive the governing differential equations of motion and a classical finite element solution is developed. As can be observed from equations (1) and (2) below, the system is coupled by the end-moments, $M$. The end-moments act about the z-axis (lagwise), however, bending in the $x-y$ plane (lagwise) is not considered and bending occurs in the $x-z$ plane (flapwise). Thus, the end-moments acting in the lagwise direction introduces torsion to the system and creates flexural-torsional coupling. Figure 1 (below) illustrates the geometry of the studied system.

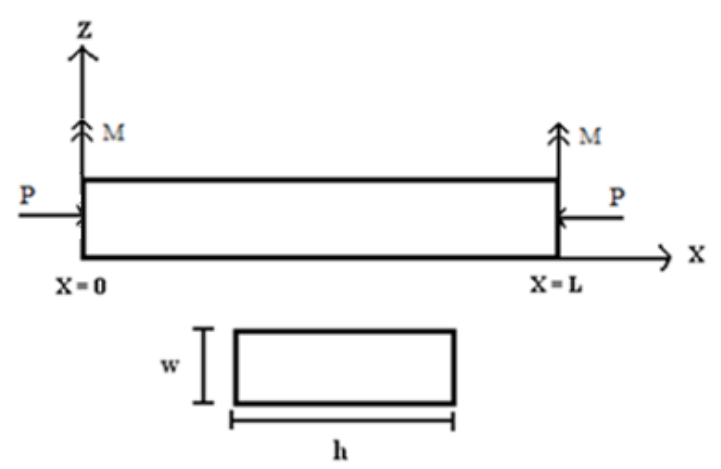

Figure 1: Beam with axial load and end-moment applied at $x=0$ and $x=L$

The two governing differential equations of the beam are as follows.

$$
\begin{gathered}
E I w^{\prime \prime \prime}+P w^{\prime \prime}+M \theta^{\prime \prime}-\rho A \ddot{w}=0 \\
G J \theta^{\prime \prime}+\frac{P I_{P}}{A} \theta^{\prime \prime}+M w^{\prime \prime}-\rho I_{P} \ddot{\theta}=0
\end{gathered}
$$

where $w$ stands for the transverse flexural displacement and $\theta$ represents the torsional displacement. The derivatives with respect to the length of the beam and time are denoted with a 
prime (') and a dot (.), respectively. In equations (1) and (2) the applied moment and force are shown as $M$ and $P$, respectively. $E I$ and $G J$ in the above equations are the Euler bending and St. Venant torsion stiffness terms, respectively. The cross-sectional area of the beam is denoted by $A$. The mass density is represented by $\rho$ and $I_{p}$ stands for the polar moment of inertia of the beam. In order to eliminate the time dependency in equations (1) and (2), simple harmonic vibration is considered and the following transformations are used to describe the transverse and torsional displacements.

$$
\begin{gathered}
w(x, t)=\hat{W} \sin (\omega t) \\
\theta(x, t)=\hat{\theta} \sin (\omega t)
\end{gathered}
$$

where $\omega$ is the circular frequency and $t$, is the time. $\widehat{W}$ and $\hat{\theta}$ are the transverse and torsional displacement amplitudes, respectively. Upon substituting equations (3) and (4), equations (1) and (2) becomes,

$$
\begin{gathered}
E I \hat{W}^{\prime \prime \prime}+P \hat{W}^{\prime \prime}+M \hat{\theta}^{\prime \prime}-\rho A \omega^{2} \hat{W}=0 \\
G J \hat{\theta}^{\prime \prime}+P I_{P} \hat{\theta}^{\prime \prime}+M \hat{W}^{\prime \prime}-\rho I_{P} A \omega^{2} \hat{\theta}=0
\end{gathered}
$$

The Galerkin method of weighted residuals [40] is employed to develop the integral form of the above equations.

$$
\begin{aligned}
& \bar{W}_{f}=\int_{0}^{L} \delta W\left(E I W^{\prime \prime \prime}+P W^{\prime \prime}+M \theta^{\prime \prime}+\rho A \omega^{2} W\right) d x=0 \\
& \bar{W}_{t}=\int_{0}^{L} \delta \theta\left(G J \theta^{\prime \prime}+\frac{P I_{P}}{A} \theta^{\prime \prime}+M W^{\prime \prime}+\rho I_{P} \omega^{2} \theta\right) d x=0
\end{aligned}
$$


where $\delta W$ and $\delta \theta$ (i.e. weighting functions) represent the transverse and torsional virtual displacements, respectively. Performing integration by parts on equations (7) and (8) leads to the weak integral form of the governing equations, written as:

$\bar{W}_{f}=\int_{0}^{L}\left(E I W^{\prime \prime} \delta W^{\prime \prime}-P W^{\prime} \delta W^{\prime}+M \theta^{\prime} \delta W^{\prime}+\rho A \omega^{2} W \delta W\right) d x+\left[\left(E I W^{\prime \prime \prime}+P W^{\prime}+M \theta^{\prime}\right) \delta W\right]_{0}^{L}-\left[\left(E I W^{\prime \prime}\right) \delta W^{\prime}\right]_{0}^{L}=0$

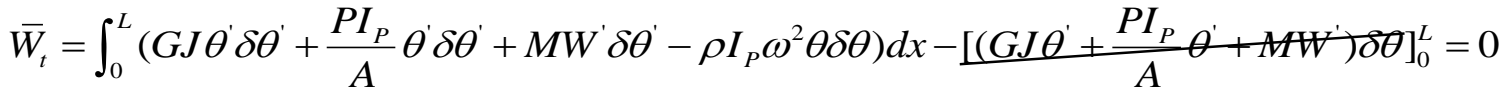

Expressions (9) and (10) also satisfy the principle of virtual work.

$$
\bar{W}=\bar{W}_{I N T}-\bar{W}_{E X T}=0
$$

where,

$$
\bar{W}_{E X T}=0
$$

and thus,

$$
\bar{W}_{I N T}=\bar{W}_{f}+\bar{W}_{t}
$$

The total virtual work, internal virtual work and external virtual work are denoted by $\bar{W}, \bar{W}_{I N T}$ and $\bar{W}_{E X T}$, respectively. The resulting shear force $S(x)$, bending moment $M(x)$, and torsional torque $T(x)$, defined as:

$$
M(x)=-E I W^{\prime \prime}
$$




$$
\begin{gathered}
S(x)=E I W^{\prime \prime \prime}+M \theta^{\prime}+P W^{\prime} \\
T(x)=G J \theta^{\prime}+\frac{P I_{P}}{A} \theta^{\prime}+M W^{\prime}
\end{gathered}
$$

are zero at the free end and the displacements are set to zero at the fixed boundaries. As a result, the bracketed boundary terms in expressions (9) and (10) vanish for all boundary conditions. The system is then discretized using elements with 2 nodes and three DOF per node as shown in Figure 2 below such that,

$$
\bar{W}=\bar{W}_{I N T}=\sum_{k=1}^{\text {No.ofElements }} \bar{W}^{k}=\sum_{k=1}^{\text {No.ofElements }} \bar{W}_{f}^{k}+\bar{W}_{t}^{k}
$$

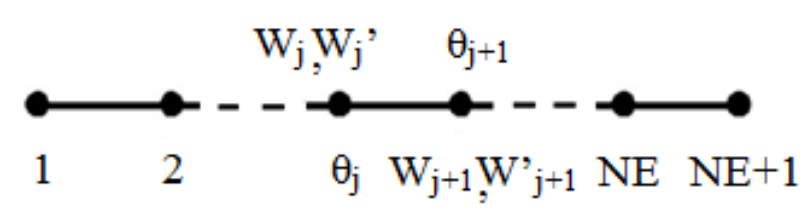

Figure 2: System discretized using elements with 3 degrees of freedom per node

Nodal DOF's are lateral displacement $w$, rotation (i.e. slope) $w$ ' and torsional displacement $\theta$. The classical finite element formulation is developed using cubic Hermite type polynomial approximations for bending displacement (equation 18) and linear approximations for torsional displacements (equation 19) introduced in the weak integral form of the governing equations such that for a two node, three degree-of-freedom per node element,

$$
\begin{gathered}
w(x)=\left\langle\begin{array}{cccc}
1 & x & x^{2} & x^{3}
\end{array}\right)\left\{C_{1}\right\} \\
\theta(x)=\langle 1 \quad x\rangle\left\{C_{2}\right\}
\end{gathered}
$$


In equations (18) and (19) above $C_{1}$ and $C_{2}$ are columns vectors of unknown constant coefficients. The vectors of nodal displacement for bending and torsion are shown below.

$$
\begin{gathered}
\left\{W_{n}\right\}=\left\{\begin{array}{l}
W_{1} \\
W_{1}^{\prime} \\
W_{2} \\
W_{2}^{\prime}
\end{array}\right\}=\left[\begin{array}{cccc}
1 & 0 & 0 & 0 \\
0 & 1 & 0 & 0 \\
1 & l & l^{2} & l^{3} \\
0 & 1 & 2 l & 3 l^{2}
\end{array}\right]\left\{C_{1}\right\}=\left[P_{n, w}\right]\left\{C_{1}\right\} \\
\left\{\theta_{n}\right\}=\left\{\begin{array}{l}
\theta_{1} \\
\theta_{2}
\end{array}\right\}=\left[\begin{array}{ll}
1 & 0 \\
1 & l
\end{array}\right]\left\{C_{2}\right\}=\left[P_{n, t}\right]\left\{C_{2}\right\}
\end{gathered}
$$

Thus,

$$
W(x)=\left\langle 1 \quad x \quad x^{2} \quad x^{3}\right\rangle\left[P_{n, w}\right]^{-1}\left\{W_{n}\right\}=\langle N(x)\rangle\left\{W_{n}\right\}
$$

and

$$
\theta(x)=\langle 1 \quad x\rangle\left[P_{n, t}\right]^{-1}\left\{\theta_{n}\right\}=\langle L(x)\rangle\left\{\theta_{n}\right\}
$$

where $\langle N(x)\rangle$ and $\langle L(x)\rangle$ are both row vectors consisting of cubic and linear shape functions for bending and torsion, respectively. The cubic shape functions $N_{1}, N_{2}, N_{3}$ and $N_{4}$ are,

$$
\begin{gathered}
N_{1}(x)=\frac{2 x^{3}}{l^{3}}-\frac{3 x^{2}}{l^{2}}+1 \\
N_{2}(x)=\frac{x^{3}}{l^{2}}-\frac{2 x^{2}}{l}+x \\
N_{3}(x)=\frac{-2 x^{3}}{l^{3}}+\frac{3 x^{2}}{l^{2}} \\
N_{4}(x)=\frac{x^{3}}{l^{2}}-\frac{x^{2}}{l}
\end{gathered}
$$


The linear shape functions $L_{1}$ and $L_{2}$ are defined as,

$$
\begin{gathered}
L_{1}(x)=1-\frac{x}{l} \\
L_{2}(x)=\frac{x}{l}
\end{gathered}
$$

A graphical representation of the shape functions described in equations (24) and (25) above could be seen in Figure 22 in Appendix A. This discretizing process leads to the element stiffness, mass and coupling matrices which when assembled together within the FEM code written in MATLAB would result in the linear Eigenvalue problem shown in equation (26) below.

$$
\begin{gathered}
\left\langle\delta W_{n}\right\rangle\left(K-\omega^{2} M\right)\left\{W_{n}\right\}=0 \\
\operatorname{det}\left(K-\omega^{2} M\right)=0
\end{gathered}
$$

where $K$ stands for the global stiffness matrix, which is a collection of all the element stiffness matrices. The global mass matrix is symbolized by $M$.

Matrix (26-a) shown below is the element mass matrix, $[m]^{k}$, and matrices (26-b) through (26-f) are the uncoupled, coupled and geometric element stiffness matrices. When matrices (26-b) through (26-f) are assembled together, the final element stiffness matrix would result. This is shown as matrix (26-g). 


$$
[m]^{k}=\left[\begin{array}{cccccc}
\frac{156 m l}{420} & \frac{22 m l^{2}}{420} & 0 & \frac{54 m l}{420} & \frac{-13 m l^{2}}{420} & 0 \\
& \frac{4 m l^{3}}{420} & 0 & \frac{13 m l^{2}}{420} & \frac{-3 m l^{3}}{420} & 0 \\
& & \frac{\rho I_{P} l}{3} & 0 & 0 & \frac{\rho I_{P} l}{6} \\
& & \frac{156 m l}{420} & \frac{-22 m l^{2}}{420} & 0 \\
& & & \frac{4 m l^{3}}{420} & 0 \\
& & & & \frac{\rho I_{P} l}{3}
\end{array}\right]
$$

where $l$ stands for the element length and $m$ represents the element mass per unit length. The element uncoupled bending stiffness matrix, $\left[k_{B}\right]$, is shown below.

$$
\left[k_{B}\right]=\left[\begin{array}{cccc}
\frac{12 E I}{l^{3}} & \frac{6 E I}{l^{2}} & \frac{-12 E I}{l^{3}} & \frac{6 E I}{l^{2}} \\
\frac{4 E I}{l} & \frac{-6 E I}{l^{2}} & \frac{2 E I}{l} \\
& \text { Sym. } & \frac{12 E I}{l^{3}} & \frac{-6 E I}{l^{2}} \\
& & & \frac{4 E I}{l}
\end{array}\right]
$$

The final element stiffness matrix is modified due to the presence of the end-moment and axial load which contributes the $[k]_{\text {geometric }}$ matrix, $[k]_{\text {torsion }}$ matrix, bending - torsion coupling stiffness matrix, $\left[k_{B T}\right]_{c}$, and the torsion - bending coupling stiffness matrix, $\left[k_{T B}\right]_{c}$. These are added to the bending stiffness matrix, $\left[k_{B}\right]$, above, to form the final element stiffness matrix, $[k]^{k}$. The geometric and torsion stiffness matrices contributed by the axial load $P$ are shown below. 


$$
\begin{gathered}
{[k]_{\text {geometric }}=P\left[\begin{array}{cccc}
\frac{6}{5 l} & \frac{1}{10} & \frac{-6}{5 l} & \frac{1}{10} \\
& \frac{2 l}{15} & \frac{-1}{10} & \frac{-l}{30} \\
& \text { Sym. } & \frac{6}{5 l} & \frac{-1}{10} \\
& & \frac{2 l}{15}
\end{array}\right]} \\
{[k]_{\text {torsion }}=\left(\frac{G J}{l}+\frac{P I_{P}}{A l}\right)\left[\begin{array}{cc}
1 & -1 \\
-1 & 1
\end{array}\right]}
\end{gathered}
$$

The bending - torsion and torsion - bending coupling stiffness matrices introduced by the endmoment $M$ are as follows.

$$
\begin{gathered}
{\left[k_{B T}\right]_{C}=\frac{M}{l}\left[\begin{array}{cccc}
1 & 0 & -1 & 0 \\
-1 & 0 & 1 & 0
\end{array}\right]} \\
{\left[k_{T B}\right]_{c}=\frac{M}{l}\left[\begin{array}{cc}
1 & -1 \\
0 & 0 \\
-1 & 1 \\
0 & 0
\end{array}\right]}
\end{gathered}
$$

Therefore, the final element stiffness matrix, which is a collection of the five sub matrices, takes the following form. 


$$
[k]^{k}=\left[\begin{array}{cccccc}
\frac{12 E I}{l^{3}}-\frac{6 P}{5 l} & \frac{6 E I}{l^{2}}-\frac{P}{10} & \frac{M}{l} & \frac{-12 E I}{l^{3}}+\frac{6 P}{5 l} & \frac{6 E I}{l^{2}}-\frac{P}{10} & \frac{-M}{l} \\
\frac{4 E I}{l}-\frac{2 P l}{15} & 0 & \frac{-6 E I}{l^{2}}+\frac{P}{10} & \frac{2 E I}{l}+\frac{P l}{30} & 0 \\
& \text { Sym. } & \frac{G J}{l}+\frac{P I_{P}}{A l} & \frac{-M}{l} & 0 & \frac{-G J}{l}-\frac{P I_{P}}{A l} \\
& & \frac{12 E I}{l^{3}}-\frac{6 P}{5 l} & \frac{-6 E I}{l^{2}}+\frac{P}{10} & \frac{M}{l} \\
& & & \frac{4 E I}{l}-\frac{2 P l}{15} & 0 \\
& & & \frac{G J}{l}+\frac{P I_{P}}{A l}
\end{array}\right]
$$

The solution to the linear Eigenvalue problem in equation (26) is achieved by determining the Eigenvalues and Eigenvectors using a FEM code developed in MATLAB. Various classical boundary conditions are also applied within the MATLAB code. Thus, the natural frequencies and mode shapes of the beam are evaluated. 


\subsection{FEM Numerical Tests}

A Steel beam ( $E=200 \mathrm{GPa}$ and $\left.\mathrm{d}=7800 \mathrm{~kg} / \mathrm{m}^{3}\right)$, having a length of $8 \mathrm{~m}$, width of $0.4 \mathrm{~m}$ and depth of $0.2 \mathrm{~m}$ was studied. The first stage of the numerical tests was to validate the results obtained using the developed FEM code with known exact results. Due to the lack of analytical results for the problem containing an axial load and end-moment, the accuracy of the natural frequency values from the code were compared with the analytical results for a beam without any force or moment. Table 1 below include the results for the first three natural frequencies for various boundary condition types using the exact [40] and FEM methods.

Table 1: Comparison between the exact and FEM results for the first three natural frequencies at $P=0$ and $M=0$

\begin{tabular}{|c|c|c|c|c|c|c|}
\hline \multirow{2}{*}{$\begin{array}{c}\text { Boundary } \\
\text { Condition }\end{array}$} & \multicolumn{6}{|c|}{ Natural Frequencies (Hz) at P $=0$ and M $=0$} \\
\cline { 2 - 7 } & \multicolumn{2}{|c|}{ Mode 1 } & \multicolumn{2}{c|}{ Mode 2 } & \multicolumn{2}{c|}{ Mode 3 } \\
\cline { 2 - 7 } & $\begin{array}{c}\text { Exact } \\
{[40]}\end{array}$ & $\begin{array}{c}\text { FEM (40 } \\
\text { elements) }\end{array}$ & $\begin{array}{c}\text { Exact } \\
{[40]}\end{array}$ & $\begin{array}{c}\text { FEM } \\
\text { (40elements) }\end{array}$ & $\begin{array}{c}\text { Exact } \\
{[40]}\end{array}$ & $\begin{array}{c}\text { FEM } \\
\text { (40elements) }\end{array}$ \\
\hline C - F & 2.556 & 2.556 & 15.995 & 15.995 & 44.858 & 44.858 \\
\hline C - C & 16.266 & 16.266 & 44.858 & 44.858 & 87.970 & 87.970 \\
\hline P - P & 7.175 & 7.175 & 28.718 & 28.718 & 64.633 & 64.633 \\
\hline P - C & 11.209 & 11.209 & 36.351 & 36.351 & 75.611 & 75.611 \\
\hline
\end{tabular}

From Table 1 above it can be observed that the results produced by the exact method and the FEM method are identical and as such the FEM code generates accurate results.

In order to determine the rate of convergence of the FEM method, the fifth natural frequency of a cantilevered beam was used. Since coupling caused by end-moment introduces more error to the system, the highest possible loading configuration with $1.85 \mathrm{MN}$ of tensile force and 9.21 MN.m of end-moment was applied. The convergence was verified by using different numbers of elements 
to generate the mesh and finding the least number of elements that would yield acceptable results. Once again, as there were no analytical results available for similar cases, the FEM code was used to determine the exact results by progressively increasing the number of elements used from 10 to 1000. Table 2 below includes these results and it can be observed that the value for the fifth natural frequency remains at $88.243 \mathrm{~Hz}$ when 200 or more elements are used. Thus, as the result has converged and it does not change even when 1000 elements are utilized, $88.243 \mathrm{~Hz}$ is taken to be the exact value for the $5^{\text {th }}$ natural frequency when the cantilevered beam is subjected to a tensile force of 1.85 MN and end-moment of 9.21 MN.m. However, since 40 elements is sufficient to obtain a result with an error less than 0.01 percent, 40 elements were considered as a reasonable number of elements for the FEM method. Convergence of the results for the first four fundamental frequencies were also checked and it was observed that these results converged to the analytical result with lesser number of elements.

Table 2: Convergence of the fifth natural frequency

\begin{tabular}{|c|c|}
\hline $\begin{array}{c}\text { No. of } \\
\text { Elements }\end{array}$ & Mode 5 (Hz) \\
\hline 10 & 88.376 \\
\hline 20 & 88.261 \\
\hline 30 & 88.255 \\
\hline 40 & 88.247 \\
\hline 50 & 88.245 \\
\hline 75 & 88.244 \\
\hline 80 & 88.244 \\
\hline 200 & 88.243 \\
\hline 500 & 88.243 \\
\hline 1000 & 88.243 \\
\hline
\end{tabular}


Figure 3 below is a graphical representation of the reduction in percentage error between the FEM and exact results as the number of elements is increased. It illustrates that the result for the fifth natural frequency of the beam subjected to the above mentioned loading and boundary conditions would converge to the exact result when 200 elements are used. It also depicts that 40 elements are sufficient to attain a percentage error below 0.01 percent.

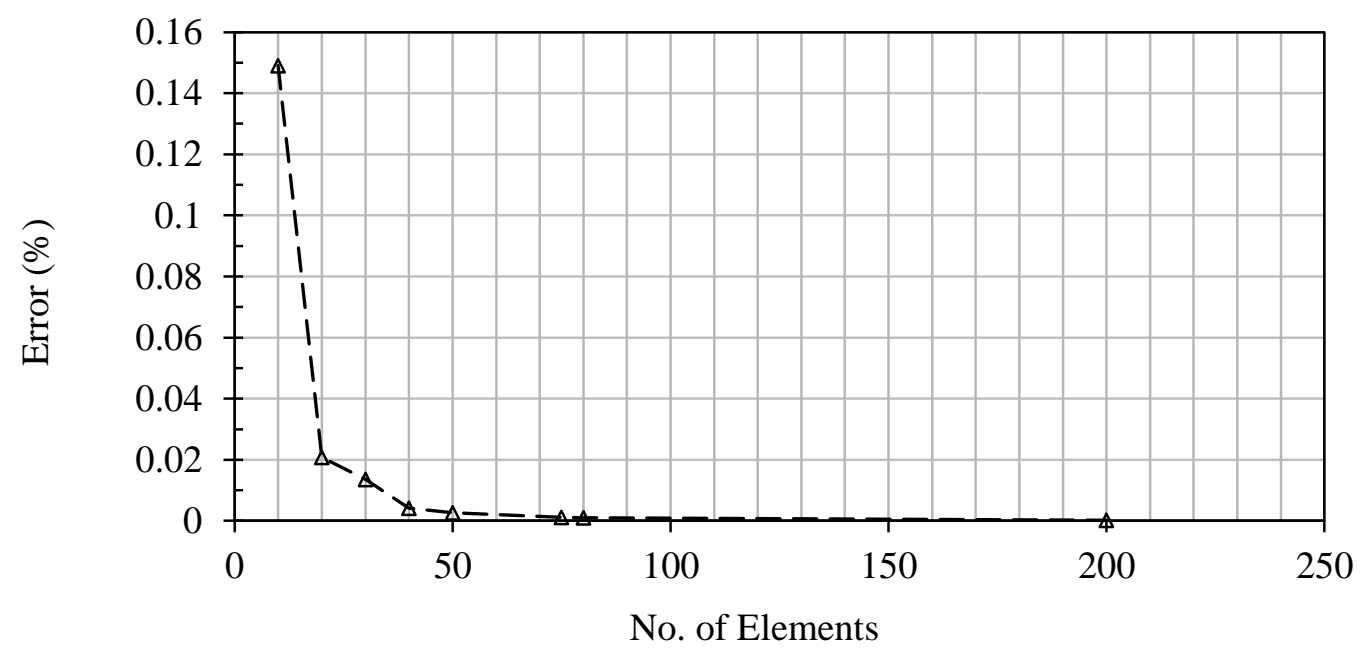

Figure 3: Convergence analysis for conventional FEM for cantilevered beam

A pre-stressed modal analysis was conducted using ANSYS 14 to simulate the problem and to further validate the FEM code results. For FEM meshing of the beam, SOLID 187 elements were used (see Figure 30 in Appendix B). The SOLID 187 element is a higher order, 3D, 10 - node element capable of 6 degrees of freedom ( 3 translations and 3 rotations) per node. A total of 102 elements were used for the meshing process in ANSYS.

The first natural frequency of the beam was determined for all classical boundary conditions when subjected to an axial tensile load and an end-moment. The accuracy of the results produced by the proposed FEM method was checked using ANSYS commercial software for the cantilevered case and the results are included in Table 3 below. 
Table 3: First natural frequencies for cantilever boundary condition $(C-F)$ when force and moment are applied; a 40-element FEM model is used

\begin{tabular}{|c|c|c|c|c|c|c|}
\hline \multirow{2}{*}{ C-F } & \multicolumn{5}{|c|}{ End-Moment } \\
\cline { 2 - 7 } & \multicolumn{5}{|c|}{0 (MN.m) } & \multicolumn{5}{|c|}{6.14 (MN.m) } & \multicolumn{2}{|c|}{9.21 (MN.m) } \\
\cline { 2 - 7 } & \multicolumn{5}{|c|}{ Fundamental Frequency (Hz) } \\
\hline $\begin{array}{c}\text { Force } \\
(\mathrm{MN})\end{array}$ & ANSYS & $\begin{array}{c}\text { FEM Code } \\
(40 \text { elements) }\end{array}$ & ANSYS & $\begin{array}{c}\text { FEM Code } \\
(40 \text { elements) }\end{array}$ & ANSYS & $\begin{array}{c}\text { FEM Code } \\
(40 \text { elements) }\end{array}$ \\
\hline 0 & 2.555 & 2.556 & 2.241 & 2.234 & 1.749 & 1.727 \\
\hline 0.62 & 2.883 & 2.884 & 2.620 & 2.614 & 2.233 & 2.216 \\
\hline 1.23 & 3.168 & 3.169 & 2.939 & 2.934 & 2.614 & 2.600 \\
\hline 1.85 & 3.421 & 3.422 & 3.217 & 3.213 & 2.934 & 2.922 \\
\hline
\end{tabular}

As can be observed from Table 3, the developed FEM code yields results that are closer to the exact results, compared to the ANSYS simulation. This could be due to the shear and warping effects of the 3D element (SOLID 187) used in ANSYS that were not accounted for in the code.

Tables 4 through 6 below include the results for the first natural frequency for different combinations of tensile force and end-moment for the clamped - clamped, pinned - pinned and pinned - clamped boundary conditions, respectively. 
Table 4: Fundamental frequencies for clamped - clamped boundary condition $(C-C)$, when force and end-moment are applied; a 40-element FEM model is used

\begin{tabular}{|c|c|c|c|}
\hline \multirow{2}{*}{ C-C } & \multicolumn{3}{|c|}{ End-Moment } \\
\cline { 2 - 4 } & 0 & 6.14 & 9.21 \\
$($ MN.m $)$ & (MN.m) & (MN.m) \\
\hline $\begin{array}{c}\text { Force } \\
(\mathrm{MN})\end{array}$ & \multicolumn{3}{|c|}{ FEM Fundamental Frequency } \\
\hline 0 & 16.266 & 16.141 & 15.984 \\
\hline 0.62 & 16.413 & 16.290 & 16.134 \\
\hline 1.23 & 16.559 & 16.437 & 16.283 \\
\hline 1.85 & 16.703 & 16.582 & 16.430 \\
\hline
\end{tabular}

Table 5: First natural frequencies for pinned - pinned boundary condition $(P-P)$ when force and moment are applied; a 40-element FEM model is used

\begin{tabular}{|c|c|c|c|}
\hline \multirow{2}{*}{ P-P } & \multicolumn{3}{|c|}{ End-Moment } \\
\cline { 2 - 4 } & 0 & 6.14 & 9.21 \\
& $($ MN.m) & (MN.m) & (MN.m) \\
\hline $\begin{array}{c}\text { Force } \\
(\mathrm{MN})\end{array}$ & \multicolumn{3}{|c|}{ FEM Fundamental Frequency } \\
\hline 0 & $7.175)$ \\
\hline 0.62 & 7.440 & 6.947 & 6.651 \\
\hline 1.23 & 7.695 & 7.483 & 7.208 \\
\hline 1.85 & 7.942 & 7.736 & 7.471 \\
\hline
\end{tabular}


Table 6: First natural frequencies for pinned - clamped boundary condition $(P-C)$ when force and moment are applied; a 40-element FEM model is used

\begin{tabular}{|c|c|c|c|}
\hline \multirow{2}{*}{ P-C } & \multicolumn{3}{|c|}{ End-Moment } \\
\cline { 2 - 4 } & 0 & 6.14 & 9.21 \\
& (MN.m) & (MN.m) & (MN.m) \\
\hline $\begin{array}{c}\text { Force } \\
(\mathrm{MN})\end{array}$ & \multicolumn{3}{|c|}{ FEM Fundamental Frequency } \\
$(\mathrm{Hz})$ \\
\hline 0 & 11.209 & 11.040 & 10.824 \\
\hline 0.62 & 11.408 & 11.242 & 11.031 \\
\hline 1.23 & 11.604 & 11.441 & 11.233 \\
\hline 1.85 & 11.796 & 11.636 & 11.432 \\
\hline
\end{tabular}

The critical buckling end-moments and compressive forces were also determined for the cantilevered boundary condition and the results are shown in Table 7 and Table 8 below.

Table 7: Critical buckling moment for cantilevered boundary condition $(C-F)$ with varying compressive force

\begin{tabular}{|c|c|}
\hline Force (MN) & Buckling Moment (MN.m) \\
\hline-1.85 & 3.900 \\
\hline-1.23 & 7.750 \\
\hline-0.62 & 10.60 \\
\hline 0 & 12.28 \\
\hline 0.62 & 13.76 \\
\hline 1.23 & 15.57 \\
\hline 1.85 & 16.95 \\
\hline
\end{tabular}


Table 8: Critical buckling compressive force for cantilevered boundary condition $(C-F)$ with varying end-moment

\begin{tabular}{|c|c|}
\hline Moment (MN.m) & $\begin{array}{c}\text { Buckling Force } \\
(\mathrm{MN})\end{array}$ \\
\hline 0 & -2.057 \\
\hline 3.07 & -1.900 \\
\hline 6.14 & -1.750 \\
\hline 9.21 & -0.900 \\
\hline
\end{tabular}

Figures 4 through 7 illustrate the variation of the first natural frequency when both tensile force and end-moment is acting on the beam, for various classical boundary conditions.

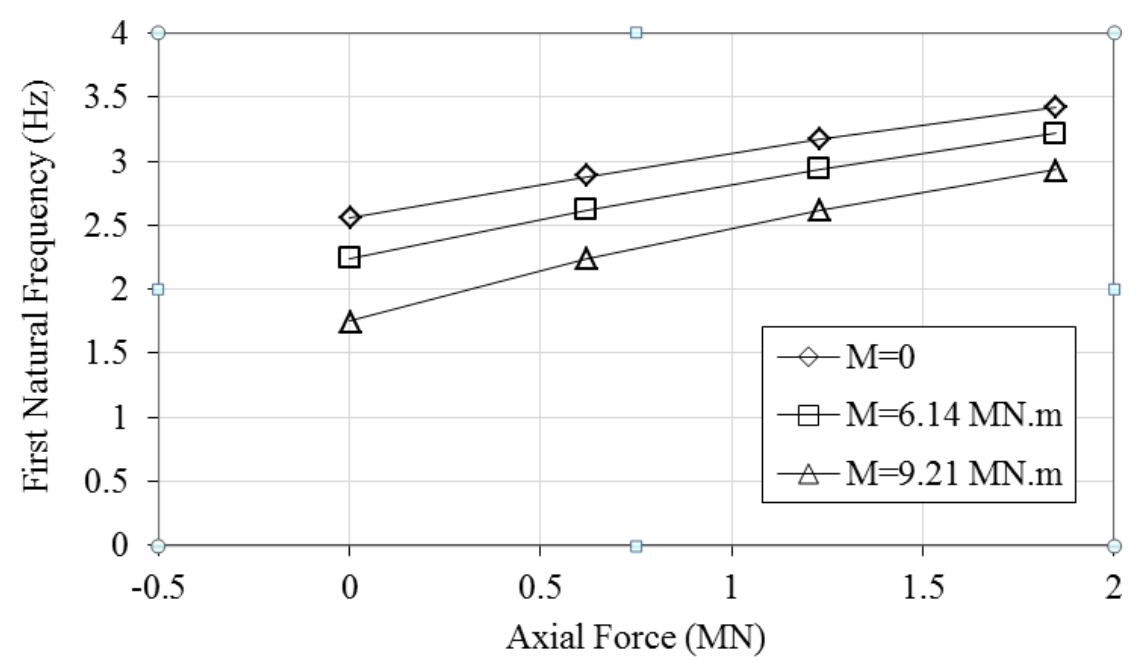

Figure 4:Variation of natural frequencies when tensile force and end-moment is applied for cantilevered (C-F) boundary condition 


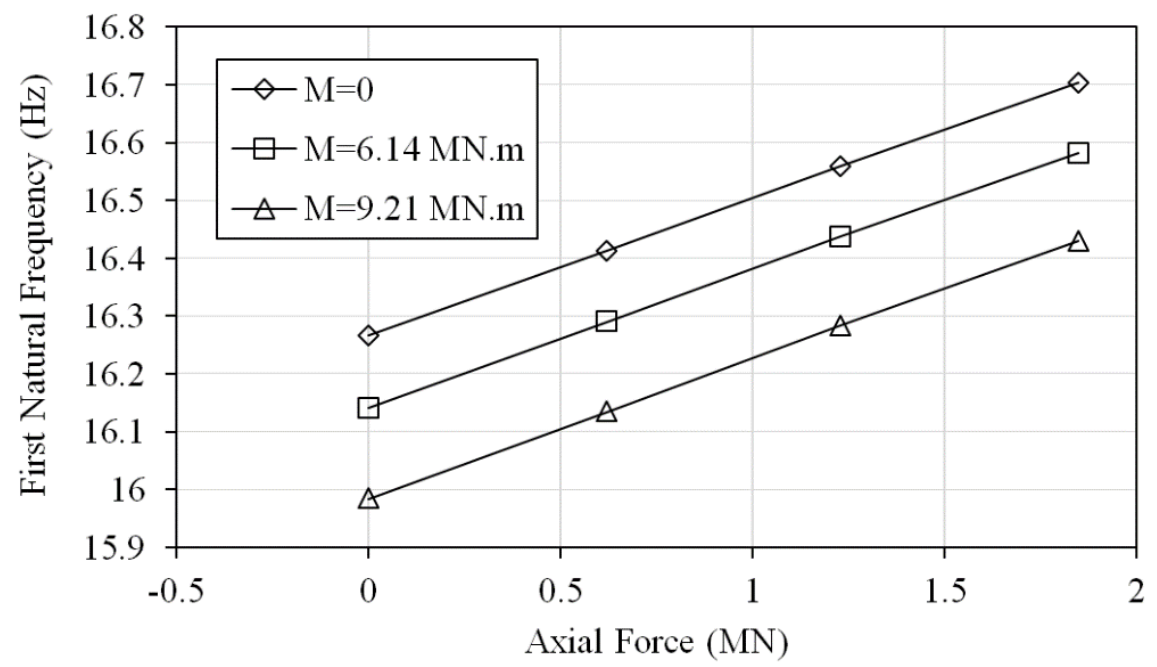

Figure 5: Variation of natural frequencies when tensile force and end-moment is applied for clampedclamped $(C-C)$ boundary condition

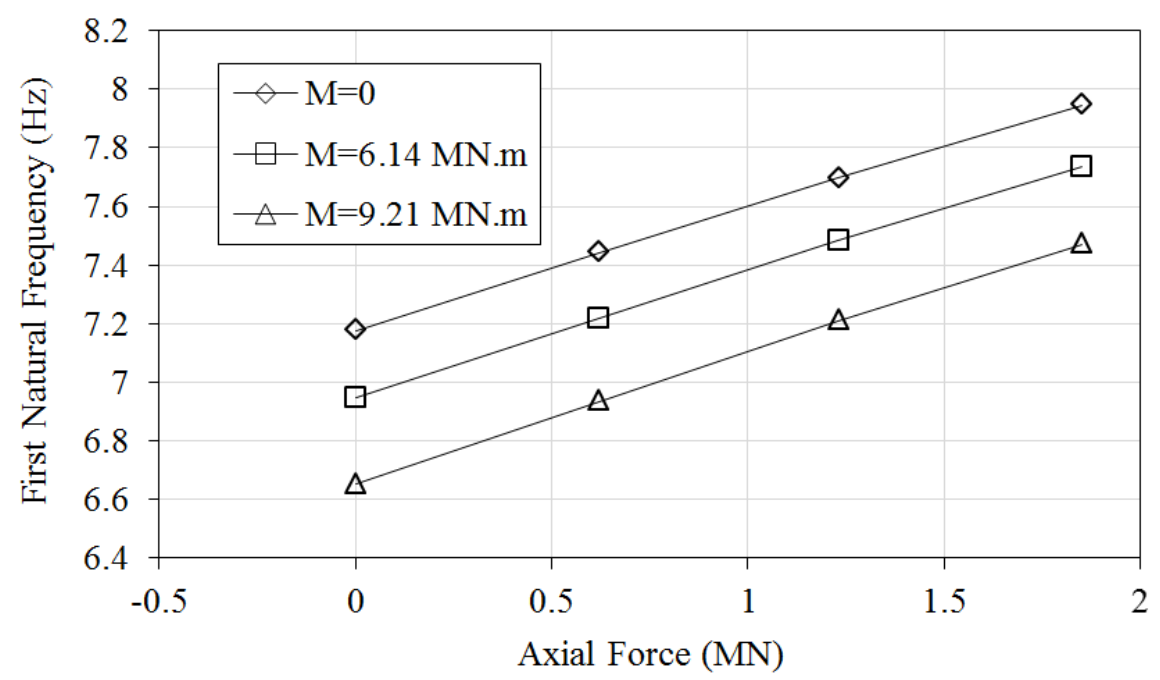

Figure 6: Variation of natural frequencies when tensile force and end-moment is applied for pinned - pinned $(P-P)$ boundary condition 


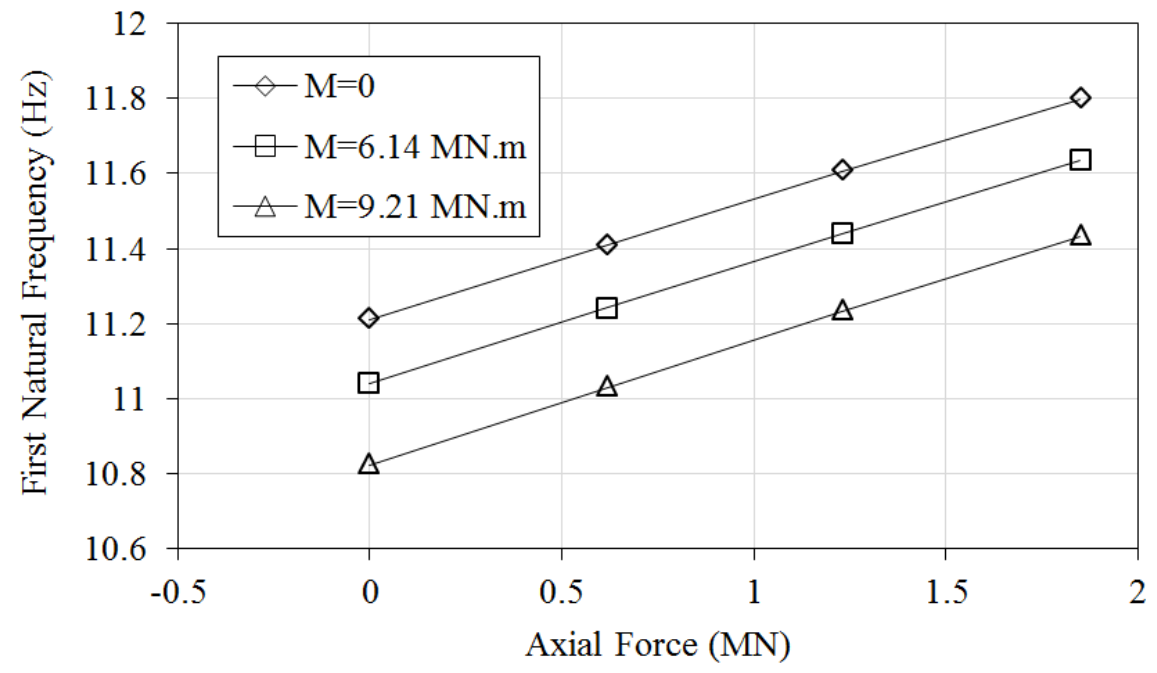

Figure 7: Variation of natural frequencies when tensile force and end-moment is applied for clamped - pinned (C-P) boundary condition

Figure 8 depicts how the critical buckling compressive force changes when the end-moment is varied. Similarly, Figure 9 shows the fluctuation of the magnitude of the critical buckling moment with respect to the change in tensile and compressive axial force.

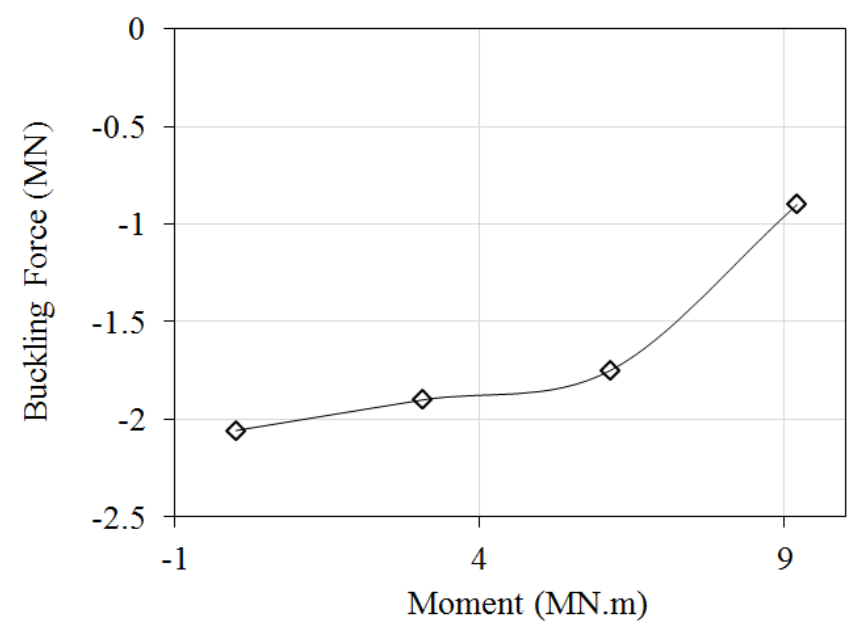

Figure 8: Variation of critical buckling compressive force with end-moment 


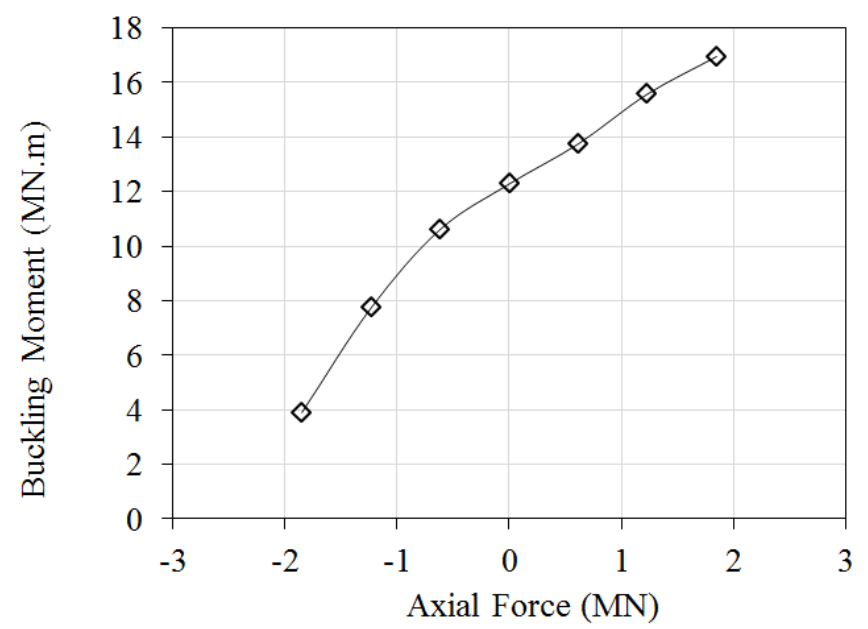

Figure 9: Variation of critical buckling end-moment with tensile and compressive force

In Figure 10 and Figure 11, respectively, the bending and torsional mode shapes of the first five natural frequencies for the cantilevered beam subjected to a tensile force of $1.85 \mathrm{MN}$ and endmoment of 9.21 MN.m are shown.

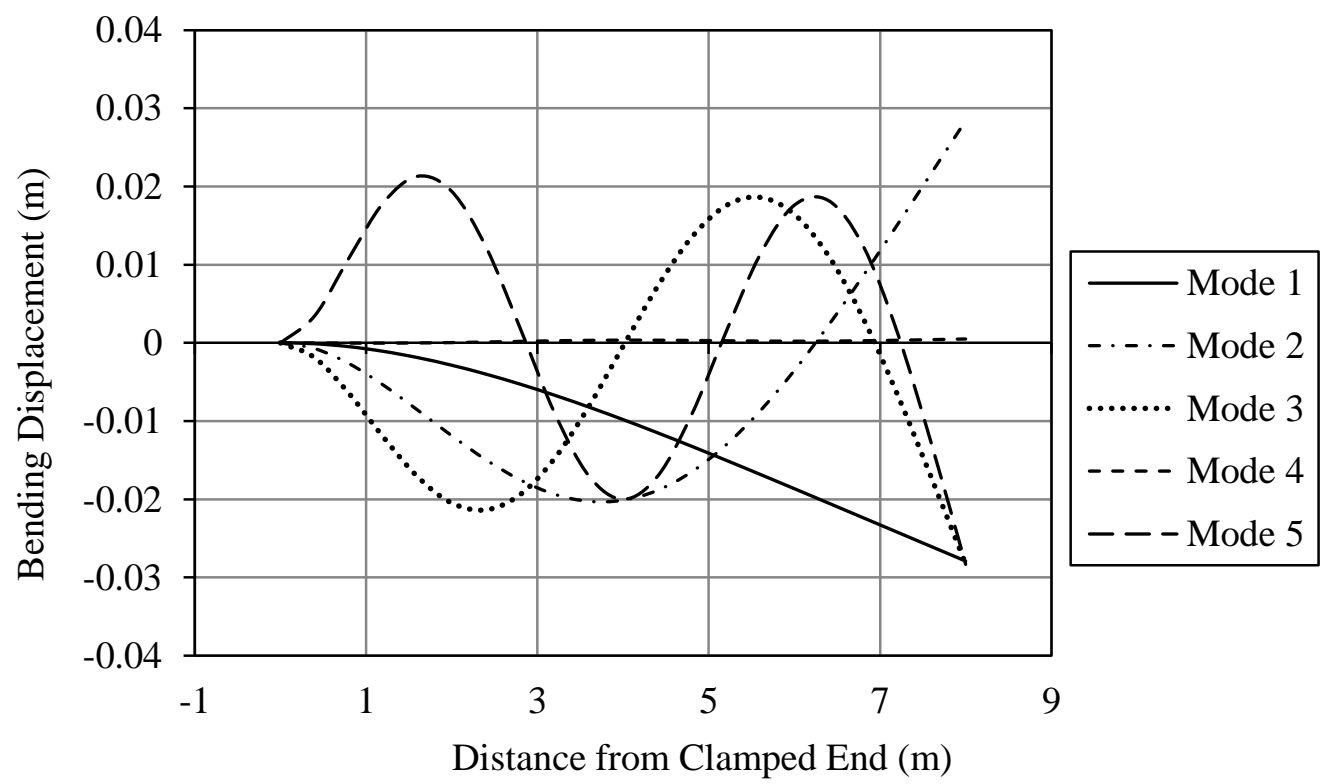

Figure 10: Bending components of mode shapes 


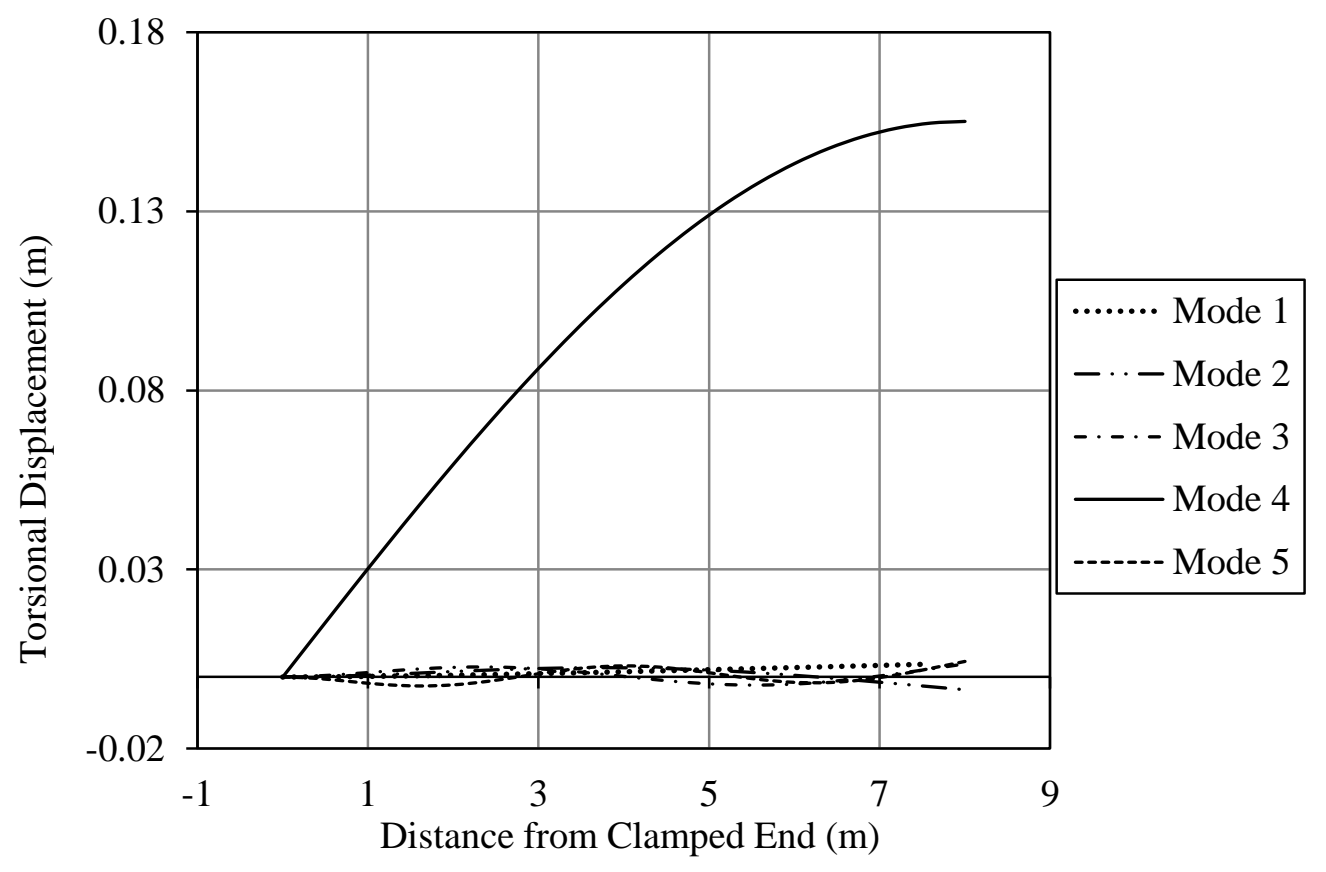

Figure 11: Torsional components of mode shapes

The frequency results of the developed FEM code showed good agreement with the ANSYS results and the error was less than 2 percent. As expected, tensile axial load increased the natural frequencies of the beam thus, indicating and increase in the stiffness of the beam for all classical boundary condition types. The increment in natural frequencies corresponding to the application of higher values of tensile load shows that the beam gets stiffer when the tensile load is increased. When the end-moment is applied without a tensile force, the natural frequencies reduce again for all boundary conditions indicating that the moment also causes a reduction in stiffness of the beam. Any increment in the magnitude of the applied end-moment lessened the stiffness of the beam further. As can be seen from Tables 3 through 6 and Figures 4 through 7, if the end-moment is held constant and the tensile load is increased, the natural frequencies increase indicating an increase in beam stiffness. However, on the contrary, if the tensile load is held constant and the end-moment is increased, the beam stiffness reduces. 
A compressive axial load has the opposite effect to that of a tensile load, on the natural frequencies and stiffness of a beam. The results in Table 7 show that the critical buckling moment is 12.28 MN.m when no force is acting, for the cantilevered boundary condition. However, when a compressive force is applied, the beam buckles at much lower magnitudes of the end-moment. Thus, the magnitude of the critical buckling moment reduces with a progressive increase in the compressive load applied. In contrast, results in Table 7 show that when a similar magnitude of tensile force is applied, the critical buckling moment increases. This further confirms the claim that tensile forces introduce additional reinforcement to the structure, increasing its stiffness. More quantitatively, the critical buckling end-moment increases from 3.9 MN.m to $16.95 \mathrm{MN} . \mathrm{m}$ simply by reversing the direction of the applied force while keeping the magnitude constant at $1.85 \mathrm{MN}$. The above results are depicted graphically in Figure 9.

Similarly, Table 8 shows that the magnitude of the critical buckling compressive force reduces with increasing values of end-moment, further confirming that the end-moment has a detrimental effect on the stiffness of the beam. This is shown in Figure 8.

Figure 10 and Figure 11, respectively, depict the flexural and torsional components of the mode shapes of the beam. As can be seen from these figures, the vibration of the beam is predominantly flexural in the first three natural frequencies. Torsion becomes predominant in the $4^{\text {th }}$ natural frequency. The $5^{\text {th }}$ natural frequency again becomes predominantly flexural. 


\section{The Dynamic Finite Element (DFE) Method}

A linearly elastic, homogeneous and isotropic beam with the same dimensions, material properties, loading configuration and boundary conditions as the beam used in the previous chapter is considered.

\subsection{The Dynamic Stiffness Matrix method}

If the beam element is considered to be uniform and homogeneous, thus, making material and geometric properties such as EI, GJ and mass uniform throughout the beam, it is possible to develop a DSM formulation. This is not always the case and material non - uniformity can make the DSM method inapplicable. However, the frequency dependent shape functions of the DFE method are found by using the exact solution to the uncoupled governing equations as expansion terms.

\subsection{The conventional FEM method based on polynomial interpolation functions}

As seen in the previous chapter, the FEM method is very flexible and convenient. Unlike the DSM method, it could be extended to include systems consisting of material and geometric non linearity making it a useful method for complex problems. However, the FEM method uses a large number of elements to achieve a reasonable degree of accuracy especially when the higher frequencies of vibration are of interest, thus, consuming more computational overhead and increasing round off error. On the other hand the DFE method has been proven in the past $[1,23]$ to produce exact results using just 1 element for uncoupled systems. Highly accurate results for coupled systems have been achieved using the DFE method with 5 or 6 elements $[1,23]$. The formulation of the equations for the FEM and DFE methods are the same up to the point of discretization (equation 17), using two-node, six degree-of-freedom elements (see Figure 2). Beyond this point, the two 
methods diverge in their formulation processes as the FEM method utilizes the cubic Hermite shape functions (equation 24) and linear shape functions (equation 25) for bending and torsion, respectively, as opposed to the DFE method which uses trigonometric shape functions presented in [1] and [23].

\subsection{The frequency- dependent Dynamic Finite Element method based on trigonometric shape}

\section{functions}

In this section, the flexural - torsional vibration and stability of a simple Euler - Bernoulli beam will be investigated using the DFE method. As mentioned previously, the DFE method is a hybrid and intermediate method that combines the accuracy of the DSM method as well as the adaptability of the conventional FEM method to obtain a better finite element model. Therefore, the starting point of the DFE formulation would be the two discretized weak form equations from the conventional FEM shown below, after the bracketed boundary terms explained in the previous chapter vanished.

$$
\begin{gathered}
\bar{W}_{f}^{k}(x)=\int_{x_{j}}^{x_{j+1}}\left(E I W^{\prime \prime} \delta W^{\prime \prime}-P W^{\prime} \delta W^{\prime}+M \theta^{\prime} \delta W^{\prime}+\rho A \omega^{2} W \delta W\right) d x \\
\bar{W}_{t}^{k}(x)=\int_{x_{j}}^{x_{j+1}}\left(G J \theta^{\prime} \delta \theta^{\prime}+\frac{P I_{P}}{A} \theta^{\prime} \delta \theta^{\prime}+M W^{\prime} \delta \theta^{\prime}-\rho I_{P} \omega^{2} \theta \delta \theta\right) d x
\end{gathered}
$$

Two sets of integration by parts will be carried out on the first two terms of the equation for flexure and one set of integration by parts will be performed on the first two terms of the equation for torsion in order to obtain the following forms.

$\bar{W}_{f}^{k}(x)=\int_{x_{j}}^{x_{j+1}}\left(E I W \delta W^{\prime \prime \prime}-P W \delta W^{\prime \prime}+\rho A \omega^{2} W \delta W\right) d x+\int_{x_{j}}^{x_{j+1}} M \theta^{\prime} \delta W^{\prime} d x+\left[E I W^{\prime} \delta W^{\prime \prime}-E I W \delta W^{\prime \prime \prime}+P W \delta W^{\prime}\right]$ 


$$
\bar{W}_{t}^{k}(x)=\int_{x_{j}}^{x_{j+1}}-\left(G J \theta \delta \theta^{\prime \prime}+\frac{P I_{p}}{A} \theta \delta \theta^{\prime \prime}+\rho I_{p} \omega^{2} \theta \delta \theta\right) d x+\int_{x_{j}}^{x_{j+1}} M W^{\prime} \delta \theta^{\prime} d x+\left[G J \theta \delta \theta^{\prime}+\frac{P I_{p}}{A} \theta \delta \theta^{\prime}\right]
$$

Substituting, $\xi=1 / l$ in both equations above would results in,

$$
\begin{gathered}
\bar{W}_{f}^{k}(\xi)=\int_{0}^{1} W(\underbrace{\frac{1}{l^{3}} E I \delta W^{\prime \prime \prime}-\frac{1}{l} P \delta W^{\prime \prime}+\rho A \omega^{2} l \delta W}_{*}) d \xi+\int_{0}^{1} \frac{1}{l} M \theta^{\prime} \delta W^{\prime} d \xi+\left[\frac{1}{l^{3}}\left[E I W^{\prime} \delta W^{\prime \prime}-E I W \delta W^{\prime \prime \prime}\right]+\frac{1}{l}\left[P W \delta W^{\prime}\right]\right] \\
\bar{W}_{t}^{k}(\xi)=\int_{0}^{1}-\theta(\underbrace{\left.\frac{1}{l} G J \delta \theta^{\prime \prime}+\frac{1}{l} \frac{P I_{p}}{A} \delta \theta^{\prime \prime}+\rho I_{p} \omega^{2} l \delta \theta\right)}_{* *} d \xi+\int_{0}^{1} \frac{1}{l} M W^{\prime} \delta \theta^{\prime} d \xi+\frac{1}{l}\left[G J \theta \delta \theta^{\prime}+\frac{P I_{p}}{A} \theta \delta \theta^{\prime}\right]
\end{gathered}
$$

The interpolation functions which respect the nodal properties would be the solutions to the integral terms marked as $(*)$ and $(* *)$. Thus, the non - nodal approximation of the solution functions, $W$ and $\theta$, and the test functions $\delta W$, and $\delta \theta$, written in terms of generalised parameters are as follows.

$$
\begin{array}{cc}
W=\langle P(\xi)\rangle_{f} *\{a\} & \delta W=\langle P(\xi)\rangle_{f} *\{\delta a\} \\
\theta=\langle P(\xi)\rangle_{t} *\{b\} & \delta \theta=\langle P(\xi)\rangle_{t} *\{\delta b\}
\end{array}
$$

The basis functions of the approximation are shown below. These basis function are the solutions to the characteristic equations $\left(^{*}\right)$ and $(* *)$. When the roots, $\alpha, \beta$, and $\tau$ of the characteristic equations tend to zero, the resulting basis functions are similar to that of a standard beam element in the classical FEM where flexure and torsion are approximated using cubic Hermite polynomials and linear functions, respectively.

$$
\begin{gathered}
\langle P(\xi)\rangle_{f}=\left\langle\cos (\alpha \xi) ; \frac{\sin (\alpha \xi)}{\alpha} ; \frac{\cosh (\beta \xi)-\cos (\alpha \xi)}{\alpha^{2}+\beta^{2}} ; \frac{\sinh (\beta \xi)-\sin (\alpha \xi)}{\alpha^{3}+\beta^{3}}\right\rangle \\
\langle P(\xi)\rangle_{t}=\langle\cos (\tau \xi) ; \sin (\tau \xi) / \tau\rangle
\end{gathered}
$$

The roots, $\alpha, \beta$, and $\tau$ are defined as follows. 


$$
X_{1}=\frac{\left\{-B+\sqrt{B^{2}-4 \bar{A} C}\right\}}{2 \bar{A}} \quad X_{2}=\frac{\left\{-B-\sqrt{B^{2}-4 \bar{A} C}\right\}}{2 \bar{A}}
$$

where the constants,

$$
\bar{A}=\frac{E I}{l^{3}} \quad B=-\left(\frac{P}{l}\right) \quad C=-\left(m l \omega^{2}\right)
$$

Thus, the roots are,

$$
\alpha=\sqrt{\left|X_{2}\right|} \quad \beta=\sqrt{\left|X_{1}\right|}
$$

and,

$$
\tau=\sqrt{\frac{\rho I_{p} \omega^{2} l^{2} A}{A G J+P I_{p}}}
$$

Replacing the generalized parameters, $\langle a\rangle,\langle\delta a\rangle,\langle b\rangle$ and $\langle\delta b\rangle$ in equations (33) and (34) with the nodal variables, $\left\langle W_{1} W_{1}^{\prime} W_{2} W_{2}^{\prime}\right\rangle,\left\langle\delta W_{1} \delta W_{1}^{\prime} \delta W_{2} \delta W_{2}^{\prime}\right\rangle,\left\langle\theta_{1} \theta_{2}\right\rangle$ and $\left\langle\delta \theta_{1} \delta \theta_{2}\right\rangle$, and re-writing equations (33) and (34) will result in equations (41) and (42) below.

$$
\begin{aligned}
\left\{W_{n}\right\} & =\left[P_{n}\right]_{f}\{a\} \\
\left\{\theta_{n}\right\} & =\left[P_{n}\right]_{t}\{b\} \quad\left\{\delta W_{n}\right\}=\left[P_{n}\right]_{f}\{\delta a\} \\
& \left\{\delta \theta_{n}\right\}=\left[P_{n}\right]_{t}\{\delta b\}
\end{aligned}
$$

The matrices, $\left[P_{n}\right]_{f}$ and $\left[P_{n}\right]_{t}$, are defined as,

$$
\left[P_{n}\right]_{f}=\left[\begin{array}{cccc}
1 & 0 & 0 & 0 \\
0 & 1 & 0 & \frac{(\beta-\alpha)}{\left(\alpha^{3}+\beta^{3}\right)} \\
\cos (\alpha) & \frac{\sin (\alpha)}{\alpha} & \frac{[\cosh (\beta)-\cos (\alpha)]}{\left(\alpha^{2}+\beta^{2}\right)} & \frac{[\sinh (\beta)-\sin (\alpha)]}{\left(\alpha^{3}+\beta^{3}\right)} \\
-\alpha \sin (\alpha) & \cos (\alpha) & \frac{[\beta \sinh (\beta)+\alpha \sin (\alpha)]}{\left(\alpha^{2}+\beta^{2}\right)} & \frac{[\beta \cosh (\beta)-\alpha \cos (\alpha)]}{\left(\alpha^{3}+\beta^{3}\right)}
\end{array}\right]
$$




$$
\left[P_{n}\right]_{t}=\left[\begin{array}{cc}
1 & 0 \\
\cos (\tau) & \frac{\sin (\tau)}{\tau}
\end{array}\right]
$$

Thus, equations (33), (34) and the, $\left[P_{n}\right]_{f}$, and $\left[P_{n}\right]_{\mathrm{t}}$, matrices above could be combined in the following manner to construct nodal approximations for flexural displacement $W(\xi)$ and torsion displacement $\theta(\xi)$.

$$
\begin{gathered}
W(\xi)=\langle P(\xi)\rangle_{f}\left[P_{n}\right]_{f}^{-1}\left\{W_{n}\right\}=\langle N(\xi)\rangle_{f}\left\{W_{n}\right\} \\
\theta(\xi)=\langle P(\xi)\rangle_{t}\left[P_{n}\right]_{t}^{-1}\left\{\theta_{n}\right\}=\langle N(\xi)\rangle_{t}\left\{\theta_{n}\right\}
\end{gathered}
$$

In equations (43) and (44), $\langle N(\xi)\rangle_{f}$, and $\langle N(\xi)\rangle_{t}$, are the frequency dependent trigonometric shape functions for flexure and torsion, respectively. Equations (43) and (44) could also be re-written as,

$$
\left\{\begin{array}{c}
W(\xi) \\
\theta(\xi)
\end{array}\right\}=[N]\left\{w_{n}\right\}
$$

where,

$$
[N]=\left[\begin{array}{cccccc}
N_{1 f}(\omega) & N_{2 f}(\omega) & 0 & N_{3 f}(\omega) & N_{4 f}(\omega) & 0 \\
0 & 0 & N_{1 t}(\omega) & 0 & 0 & N_{2 t}(\omega)
\end{array}\right]
$$

and

$$
\left\{w_{n}\right\}=\left\langle W_{1} W_{1}^{\prime} \theta_{1} W_{2} W_{2}^{\prime} \theta_{2}\right\rangle^{T}
$$

The definitions of the frequency-dependent trigonometric shape functions for flexure obtained from [1] and [23] are as follows. Although, dependent on the frequency $\omega$ elsewhere (see Figures 24 through 29 in Appendix A), the trigonometric shape functions for bending are independent of the frequency at the element boundaries and as such, $N_{1 f}=1, N_{2 f}=0, N_{3 f}=0$, and $N_{4 f}=0$, at $\xi=0$ 
, and $N_{l f}=0, N_{2 f}=0, N_{3 f}=1$, and $N_{4 f}=0$, at $\xi=1$. See Figure 23 in Appendix A for an illustration of flexural shape functions used for the DFE method.

$$
\begin{aligned}
& N_{1 f}(\omega)=\frac{(\alpha \beta)}{D_{f}} *\{-\cos (\alpha \xi)+\cos (\alpha(1-\xi)) * \cosh (\beta)+\cos (\alpha) * \cosh (\beta(1-\xi))- \\
& \left.\cosh (\beta \xi)-\frac{\beta}{\alpha} * \sin (\alpha(1-\xi)) * \sinh (\beta)+\frac{\alpha}{\beta} * \sin (\alpha) * \sinh (\beta(1-\xi))\right\} \\
& N_{2 f}(\omega)=\frac{1}{D_{f}} *\{\beta *[\cosh (\beta(1-\xi)) * \sin (\alpha)-\cosh (\beta) * \sin (\alpha(1-\xi))-\sin (\alpha \xi)] \\
& +\alpha *[\cos (\alpha(1-\xi)) * \sinh (\beta)-\cos (\alpha) * \sinh (\beta(1-\xi))-\sinh (\beta \xi)]\} \\
& N_{3 f}(\omega)=\frac{(\alpha \beta)}{D_{f}} *\{-\cos (\alpha(1-\xi))+\cos (\alpha \xi) * \cosh (\beta)-\cosh (\beta(1-\xi))+\cos (\alpha) * \\
& \left.\cosh (\beta \xi)-\frac{\beta}{\alpha} * \sin (\alpha \xi) * \sinh (\beta)+\frac{\alpha}{\beta} * \sin (\alpha) * \sinh (\beta \xi)\right\} \\
& N_{4 f}(\omega)=\frac{1}{D_{f}} *\{\beta *[-\cosh (\beta \xi) * \sin (\alpha)+\sin (\alpha(1-\xi))+\cosh (\beta) * \sin (\alpha \xi)] \\
& +\alpha *[-\cos (\alpha \xi) * \sinh (\beta)+\sinh (\beta(1-\xi))+\cos (\alpha) * \sinh (\beta \xi)]\}
\end{aligned}
$$

where,

$$
D_{f}=(\alpha \beta) *\left\{-2 *(1-\cos (\alpha) * \cosh (\beta))+\left(\frac{\alpha^{2}-\beta^{2}}{\alpha \beta}\right) * \sin (\alpha) * \sinh (\beta)\right\}
$$

The trigonometric shape functions for torsion presented in [1] and [23] are shown below. Similarly, these shape functions are also independent of $\omega$ at the element boundaries such that, $N_{l t}=1$ and $N_{2 t}=0$, at $\xi=0$ and $N_{1 t}=0$ and $N_{2 t}=1$, at $\xi=1$. See Figure 23 in Appendix A for a graphical representation of the torsional shape functions utilized in the DFE method. 


$$
\begin{gathered}
N_{1 t}(\omega)=\cos (\tau \xi)-\cos (\tau) * \frac{\sin (\tau \xi)}{D_{t}} \\
N_{2 t}(\omega)=\frac{\sin (\tau \xi)}{D_{t}}
\end{gathered}
$$

where,

$$
D_{t}=\sin (\tau)
$$

Therefore, using equations (31), (32) and the shape functions (48) through (55), the element stiffness matrix is obtained. The element stiffness matrix, $\left[K_{D S}\right]^{k}$, consists of two coupled dynamic stiffness matrices, $\left[K_{D S}\right]_{B T, c}^{k}$, and $\left[K_{D S}\right]_{T B, c}^{k}$, symbolized collectively as, $\left[K_{D S}\right]_{c}^{k}$, and four uncoupled dynamic stiffness matrices, $\left[K_{D S}\right]_{u 1}^{k},\left[K_{D S}\right]_{u 2}^{k},\left[K_{D S}\right]_{u 3}^{k}$, and $\left[K_{D S}\right]_{u 4}^{k}$, jointly denoted as, $\left[K_{D S}\right]_{u}^{k}$.

The final element dynamic stiffness matrix, $\left[K_{D S}\right]^{k}$, is determined by assembling these six coupled and uncoupled sub matrices as shown below.

$$
\bar{W}^{k}=\left\{\delta w_{n}\right\}\left\{\left[K_{D S}\right]_{c}^{k}+\left[K_{D S}\right]_{u}^{k}\right\}\left\{w_{n}\right\}
$$

The global dynamic stiffness matrix, $\left[K_{D S}\right]$, is then obtained by assembling all the element stiffness matrices together. This process was performed using a DFE code written in MATLAB software which resulted in the non - linear Eigenvalue problem shown in equation 57 below.

$$
\left[K_{D S}(\omega)\right]\left\{W_{n}\right\}=0
$$

Matrices (57-a) through (57-d) below are the four uncoupled element stiffness matrices mentioned above. 


$$
\begin{gathered}
{\left[K_{D S}\right]_{u 1}^{k}=\left.\frac{E I}{L^{3}}\left[\begin{array}{llll}
N_{1 f}^{\prime} N_{1 f}^{\prime \prime} & N_{1 f}^{\prime} N_{2 f}^{\prime \prime} & N_{1 f}^{\prime} N_{3 f}^{\prime \prime} & N_{1 f}^{\prime} N_{4 f}^{\prime \prime} \\
N_{2 f}^{\prime} N_{1 f}^{\prime \prime} & N_{2 f}^{\prime} N_{2 f}^{\prime \prime} & N_{2 f}^{\prime} N_{3 f}^{\prime \prime} & N_{2 f}^{\prime} N_{4 f}^{\prime \prime} \\
N_{3 f}^{\prime} N_{1 f}^{\prime \prime} & N_{3 f}^{\prime} N_{2 f}^{\prime \prime} & N_{3 f}^{\prime} N_{3 f}^{\prime \prime} & N_{3 f}^{\prime} N_{4 f}^{\prime \prime} \\
N_{4 f}^{\prime} N_{1 f}^{\prime \prime} & N_{4 f}^{\prime} N_{2 f}^{\prime \prime} & N_{4 f}^{\prime} N_{3 f}^{\prime \prime} & N_{4 f}^{\prime} N_{4 f}^{\prime \prime}
\end{array}\right]\right|_{0} ^{1}} \\
{\left[K_{D S}\right]_{u 2}^{k}=\left.\frac{-E I}{L^{3}}\left[\begin{array}{llll}
N_{1 f} N_{1 f}^{\prime \prime \prime} & N_{1 f} N_{2 f}^{\prime \prime \prime} & N_{1 f} N_{3 f}^{\prime \prime \prime} & N_{1 f} N_{4 f}^{\prime \prime \prime} \\
N_{2 f} N_{1 f}^{\prime \prime} & N_{2 f} N_{2 f}^{\prime \prime} & N_{2 f} N_{3 f}^{\prime \prime} & N_{2 f} N_{4 f}^{\prime \prime} \\
N_{3 f} N_{1 f}^{\prime \prime \prime} & N_{3 f} N_{2 f}^{\prime \prime \prime} & N_{3 f} N_{3 f}^{\prime \prime \prime} & N_{3 f} N_{4 f}^{\prime \prime \prime} \\
N_{4 f} N_{1 f}^{\prime \prime \prime} & N_{4 f} N_{2 f}^{\prime \prime \prime} & N_{4 f} N_{3 f}^{\prime \prime \prime} & N_{4 f} N_{4 f}^{\prime \prime}
\end{array}\right]\right|_{0} ^{1}} \\
{\left[K_{D S}\right]_{u 3}^{k}=\left.\frac{P}{L}\left[\begin{array}{llll}
N_{1 f} N_{1 f}^{\prime} & N_{1 f} N_{2 f}^{\prime} & N_{1 f} N_{3 f}^{\prime} & N_{1 f} N_{4 f}^{\prime} \\
N_{2 f} N_{1 f}^{\prime} & N_{2 f} N_{2 f}^{\prime} & N_{2 f} N_{3 f}^{\prime} & N_{2 f} N_{4 f}^{\prime} \\
N_{3 f} N_{1 f}^{\prime} & N_{3 f} N_{2 f}^{\prime} & N_{3 f} N_{3 f}^{\prime} & N_{3 f} N_{4 f}^{\prime} \\
N_{4 f} N_{1 f}^{\prime} & N_{4 f} N_{2 f}^{\prime} & N_{4 f} N_{3 f}^{\prime} & N_{4 f} N_{4 f}^{\prime}
\end{array}\right]\right|_{0} ^{1}} \\
{\left[K_{D S}\right]_{u 4}^{k}=\frac{1}{L}\left(G J+\frac{P I_{P}}{G}\right)\left[\begin{array}{lll}
N_{1 t} N_{1 t}^{\prime} & N_{1 t} N_{2 t}^{\prime} \\
N_{2 t} N_{1 t}^{\prime} & N_{2 t} N_{2 t}^{\prime}
\end{array}\right]_{0}^{1}}
\end{gathered}
$$

Matrices (57-e) and (57-f) are the two coupled stiffness matrices stated previously.

$$
\begin{gathered}
{\left[K_{D S}\right]_{B T, c}^{k}=\int_{0}^{1} \frac{M}{L}\left[\begin{array}{llll}
N_{1 t}^{\prime} N_{1 f}^{\prime} & N_{1 t}^{\prime} N_{2 f}^{\prime} & N_{1 t}^{\prime} N_{3 f}^{\prime} & N_{1 t}^{\prime} N_{4 f}^{\prime} \\
N_{2 t}^{\prime} N_{1 f}^{\prime} & N_{2 t}^{\prime} N_{2 f}^{\prime} & N_{2 t}^{\prime} N_{3 f}^{\prime} & N_{2 t}^{\prime} N_{4 f}^{\prime}
\end{array}\right] d \xi} \\
{\left[K_{D S}\right]_{T B, c}^{k}=\int_{0}^{1} \frac{M}{L}\left[\begin{array}{ll}
N_{1 f}^{\prime} N_{1 t}^{\prime} & N_{1 f}^{\prime} N_{2 t}^{\prime} \\
N_{2 f}^{\prime} N_{1 t}^{\prime} & N_{2 f}^{\prime} N_{2 t}^{\prime} \\
N_{3 f}^{\prime} N_{1 t}^{\prime} & N_{3 f}^{\prime} N_{2 t}^{\prime} \\
N_{4 f}^{\prime} N_{1 t}^{\prime} & N_{4 f}^{\prime} N_{2 t}^{\prime}
\end{array}\right] d \xi}
\end{gathered}
$$

Various classical boundary conditions were also applied on the global dynamic stiffness matrix within the MATLAB code. The natural frequencies of the system would be the values of $\omega$ that would yield a zero determinant for the global dynamic stiffness matrix. This is obtained by sweeping the frequency domain using visual approximation to find particular values of $\omega$ that 
produce a zero determinant. The Eigenvectors corresponding to these natural frequencies provide the mode shapes of the system. 


\subsection{DFE Numerical Tests}

Figure 12 below depicts the convergence analysis carried out for the $5^{\text {th }}$ natural frequency of a cantilevered beam when a tensile force of 1.85 MN and end-moment of 9.21 MN.m was acting. As the DFE method yields exact results with just one element for all other loading configurations pertaining to this study, except for when an end-moment is applied, loading configurations with no moment were not considered for the convergence analysis. Furthermore, the same loading conditions used for the convergence analysis of the FEM method were utilized to ensure ease of comparison of the rates of convergence of the two methods. A comparison between the DFE method and conventional FEM with regards to the efficiency in convergence is illustrated in Figure 13. For the $5^{\text {th }}$ natural frequency, the DFE method produces results with an error less than 0.2 percent compared to the exact result with just 5 elements or to an error less than 0.1 percent using 8 elements. As can be seen from both Figure 12 and Figure 13, if the number of elements in the DFE method is increased to 11, the percentage error would be further reduced. In contrast, the FEM method requires at least 20 elements to achieve an error less than 0.1 percent for the $5^{\text {th }}$ natural frequency. Thus, even for higher natural frequencies the DFE method uses 2.5 times less elements than the FEM method and as such the former is capable of converging faster. It is important to note here that for the $1^{\text {st }}, 2^{\text {nd }}, 3^{\text {rd }}$ and $4^{\text {th }}$ natural frequencies, the DFE method produces much accurate results with even fewer elements. This can be observed from Table 9 below which shows that the DFE method converges to the FEM result for the $1^{\text {st }}$ natural frequency with an error between $0-0.17$ percent using just 5 elements, whereas the FEM method required 40 elements to achieve this. Thus, the FEM method used 8 times more elements than the DFE method.

Table 9 also shows that when no end-moment is acting, the DFE method is capable of producing the exact results [40] for the uncoupled case of $\mathrm{P}=0$ and $\mathrm{M}=0$ using just 1 element whereas the 
FEM requires 40 elements to achieve the same degree of accuracy. Since, the DFE method uses the exact solutions to the uncoupled governing differential equations as expansion terms (i.e. basis functions of approximation space) to obtain the frequency dependant shape functions that are later utilized to approximate the bending and torsional displacements as well as rotation, the DFE method is capable of producing the exact results with a single element if the system is uncoupled. However, when the end-moment is applied, the DFE method requires up to 5 elements to generate results similar to the FEM results, with an error less than 0.2 percent. According to the third terms (coupling terms) of both equations (1) and (2), the end-moment, $M$, couples the two governing differential equations. When the dynamic shape functions that are based on the solutions to the uncoupled systems are used to approximate the behaviour of a geometrically coupled system when end-moment is applied, error is introduced in to the calculations and thus, more than one element is required to converge the results to a suitable degree of accuracy.

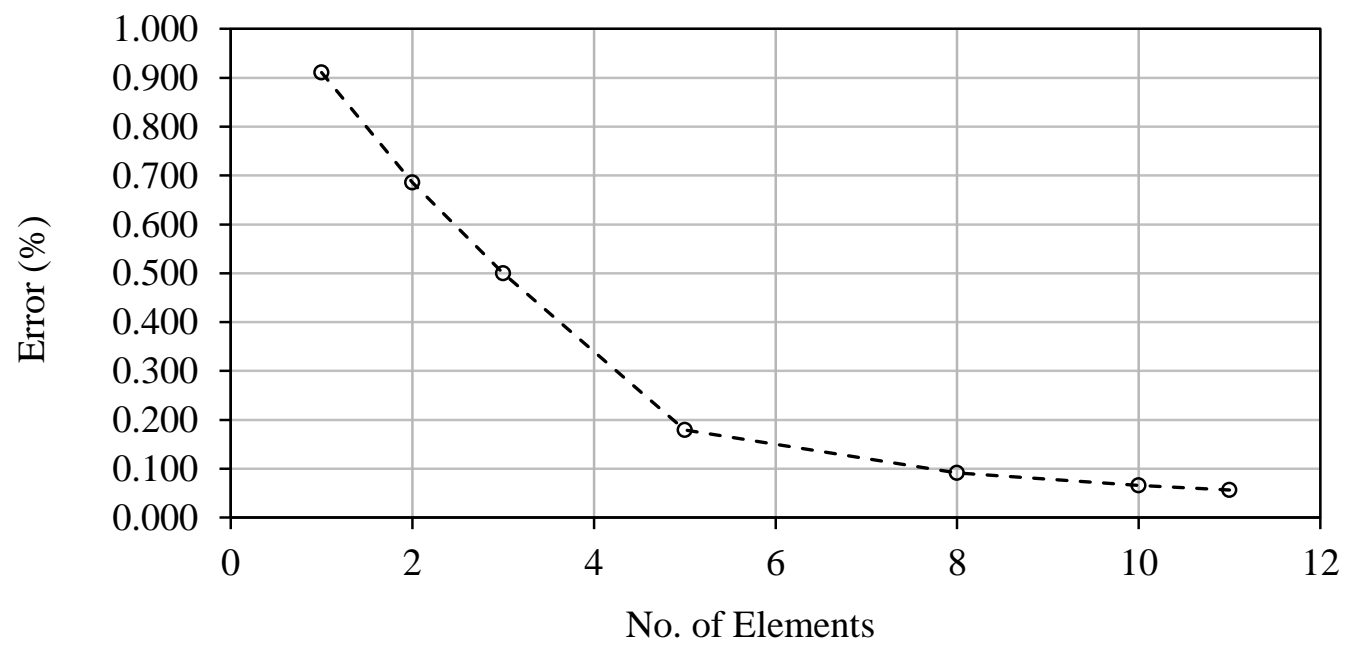

Figure 12: Convergence analysis for DFE method for cantilevered beam 


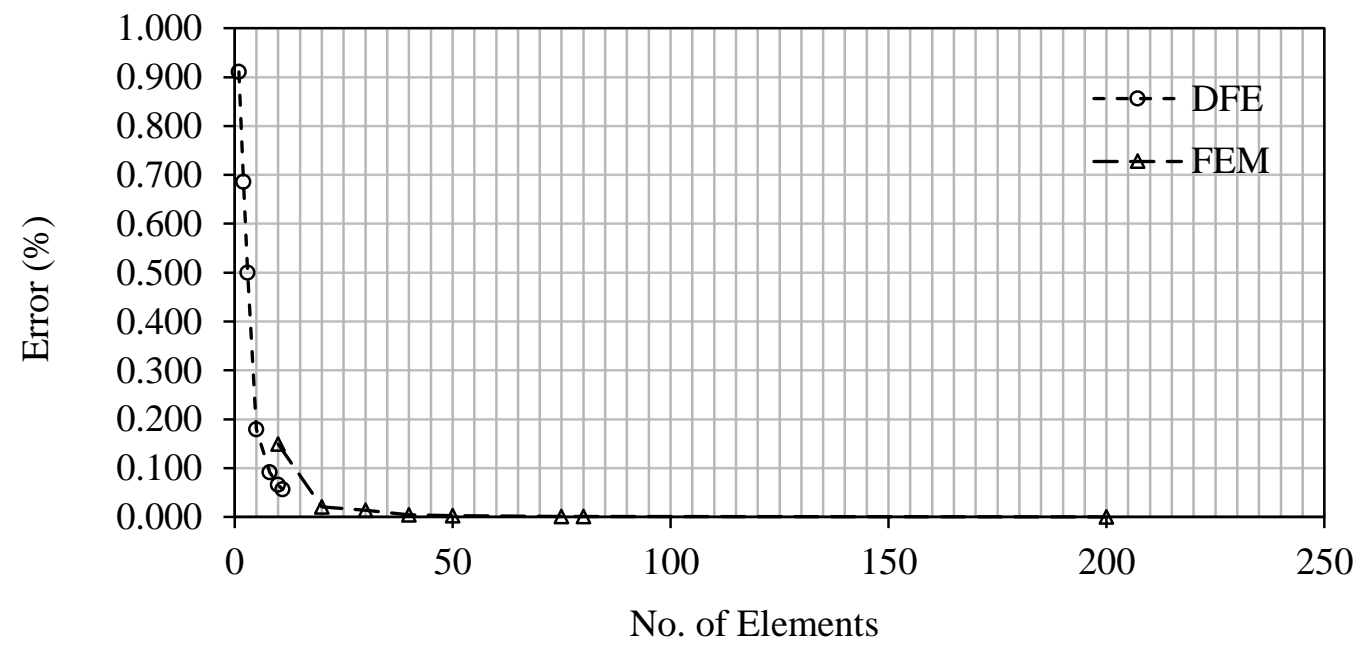

Figure 13: Comparison of convergence efficiency between DFE method and conventional FEM for cantilevered beam

Tables 9 through 12 show the results for the first natural frequency for cantilevered, clamped clamped, pinned - pinned and pinned - clamped boundary conditions, respectively.

Table 9: First natural frequencies for cantilever beam $(C-F)$ when force and moment are applied

\begin{tabular}{|c|c|c|c|c|c|c|c|c|c|}
\hline \multirow{2}{*}{ C-F } & \multicolumn{7}{|c|}{ End-Moment } \\
\cline { 2 - 9 } & \multicolumn{7}{|c|}{0 (MN.m) } & \multicolumn{7}{|c|}{6.14 (MN.m) } & \multicolumn{3}{c|}{9.21 (MN.m) } \\
\cline { 2 - 9 } & \multicolumn{7}{|c|}{ FEM Fundamental Frequency (Hz) } \\
\hline Force & $\begin{array}{c}\text { DFE (1 } \\
\text { (MN) }\end{array}$ & FEM (40 & DFE (5 & FEM (40 & Error & DFE (5 \\
elements) & elements) & FEM (40 & Error \\
elements) & $\%)$ & elements) & elements) & $(\%)$ \\
\hline 0 & 2.556 & 2.556 & 2.237 & 2.234 & 0.13 & 1.730 & 1.727 & 0.17 \\
\hline 0.62 & 2.884 & 2.884 & 2.617 & 2.614 & 0.11 & 2.219 & 2.216 & 0.14 \\
\hline 1.23 & 3.169 & 3.169 & 2.935 & 2.934 & 0.02 & 2.603 & 2.6 & 0.12 \\
\hline 1.85 & 3.422 & 3.422 & 3.213 & 3.213 & 0.00 & 2.925 & 2.922 & 0.10 \\
\hline
\end{tabular}


Table 10: First natural frequencies for clamped - clamped boundary condition $(C-C)$ when force and moment are applied

\begin{tabular}{|c|c|c|c|c|c|c|}
\hline \multirow{3}{*}{ C-C } & \multicolumn{6}{|c|}{ End-Moment } \\
\hline & \multicolumn{2}{|c|}{0 (MN.m) } & \multicolumn{2}{|c|}{ 6.14(MN.m) } & \multicolumn{2}{|c|}{ 9.21(MN.m) } \\
\hline & \multicolumn{6}{|c|}{ FEM Fundamental Frequency (Hz) } \\
\hline Force & DFE $(1$ & FEM (40 & DFE $(5$ & FEM $(40$ & DFE $(5$ & FEM $(40$ \\
\hline$(\mathrm{MN})$ & element) & elements) & elements) & elements) & elements) & elements) \\
\hline 0 & 16.266 & 16.266 & 16.157 & 16.141 & 16.019 & 15.984 \\
\hline 0.62 & 16.413 & 16.413 & 16.306 & 16.290 & 16.170 & 16.134 \\
\hline 1.23 & 16.559 & 16.559 & 16.451 & 16.437 & 16.316 & 16.283 \\
\hline 1.85 & 16.703 & 16.703 & 16.597 & 16.582 & 16.464 & 16.430 \\
\hline
\end{tabular}

Table 11: First natural frequencies for pinned - pinned boundary condition $(P-P)$ when force and moment are applied

\begin{tabular}{|c|c|c|c|c|c|c|}
\hline \multirow{2}{*}{ P-P } & \multicolumn{6}{|c|}{ End-Moment } \\
\cline { 2 - 7 } & \multicolumn{7}{|c|}{0 (MN.m) } & \multicolumn{2}{|c|}{6.14 (MN.m) } & \multicolumn{2}{c|}{9.21 (MN.m) } \\
\cline { 2 - 7 } & \multicolumn{5}{|c|}{ Fundamental Frequency (Hz) } \\
\hline $\begin{array}{c}\text { Force } \\
\text { (MN) }\end{array}$ & $\begin{array}{c}\text { DFE (1 } \\
\text { element) }\end{array}$ & $\begin{array}{c}\text { FEM (40 } \\
\text { elements) }\end{array}$ & $\begin{array}{c}\text { DFE (5 } \\
\text { element) }\end{array}$ & $\begin{array}{c}\text { FEM (40 } \\
\text { elements) }\end{array}$ & $\begin{array}{c}\text { DFE (5 } \\
\text { element) }\end{array}$ & $\begin{array}{c}\text { FEM (40 } \\
\text { elements) }\end{array}$ \\
\hline 0 & 7.175 & 7.175 & 6.955 & 6.947 & 6.669 & 6.651 \\
\hline 0.62 & 7.440 & 7.440 & 7.228 & 7.220 & 6.954 & 6.935 \\
\hline 1.23 & 7.695 & 7.695 & 7.488 & 7.483 & 7.223 & 7.208 \\
\hline 1.85 & 7.942 & 7.942 & 7.743 & 7.736 & 7.487 & 7.471 \\
\hline
\end{tabular}


Table 12: First natural frequencies for pinned - clamped boundary condition $(P-C)$ when force and moment are applied

\begin{tabular}{|c|c|c|c|c|c|c|}
\hline \multirow{3}{*}{ P-C } & \multicolumn{6}{|c|}{ End-Moment } \\
\hline & \multicolumn{2}{|c|}{0 (MN.m) } & \multicolumn{2}{|c|}{6.14 (MN.m) } & \multicolumn{2}{|c|}{9.21 (MN.m) } \\
\hline & \multicolumn{6}{|c|}{ FEM Fundamental Frequency $(\mathrm{Hz})$} \\
\hline Force & DFE $(1$ & FEM $(40$ & DFE $(5$ & FEM $(40$ & DFE $(5$ & FEM $(40$ \\
\hline$(\mathrm{MN})$ & element) & elements) & elements) & elements) & elements) & elements) \\
\hline 0 & 11.209 & 11.209 & 11.051 & 11.040 & 10.851 & 10.824 \\
\hline 0.62 & 11.408 & 11.408 & 11.254 & 11.242 & 11.058 & 11.031 \\
\hline 1.23 & 11.604 & 11.604 & 11.451 & 11.441 & 11.257 & 11.233 \\
\hline 1.85 & 11.796 & 11.796 & 11.646 & 11.636 & 11.456 & 11.432 \\
\hline
\end{tabular}

A buckling analysis was also carried out using the DFE method and the results for the critical buckling compressive force and end-moment are included in Table 13 and Table 14 below.

Table 13: Critical buckling moment for cantilevered boundary condition $(C-F)$ with varying compressive force

\begin{tabular}{|c|c|}
\hline Force (MN) & $\begin{array}{c}\text { Buckling Moment (MN.m) } \\
\text { DFE (5 element) }\end{array}$ \\
\hline-1.85 & 3.91 \\
\hline-1.23 & 7.82 \\
\hline-0.62 & 10.31 \\
\hline 0 & 12.33 \\
\hline 0.62 & 14.07 \\
\hline 1.23 & 15.59 \\
\hline 1.85 & 17.00 \\
\hline
\end{tabular}


Table 14: Critical buckling compressive force for cantilevered boundary condition $(C-F)$ with varying end-moment

\begin{tabular}{|c|c|}
\hline $\begin{array}{c}\text { Moment } \\
\text { (MN.m) }\end{array}$ & $\begin{array}{c}\text { Buckling Force (MN) } \\
\text { DFE (5 elements) }\end{array}$ \\
\hline 0 & -2.06 \\
\hline 3.07 & -1.93 \\
\hline 6.14 & -1.55 \\
\hline 9.21 & -0.91 \\
\hline
\end{tabular}

Figures 14 through 17 below are graphical representations of the results in Tables 9 through 12 . These figures illustrate the variation of the first fundamental frequency with tensile axial force and end-moment.

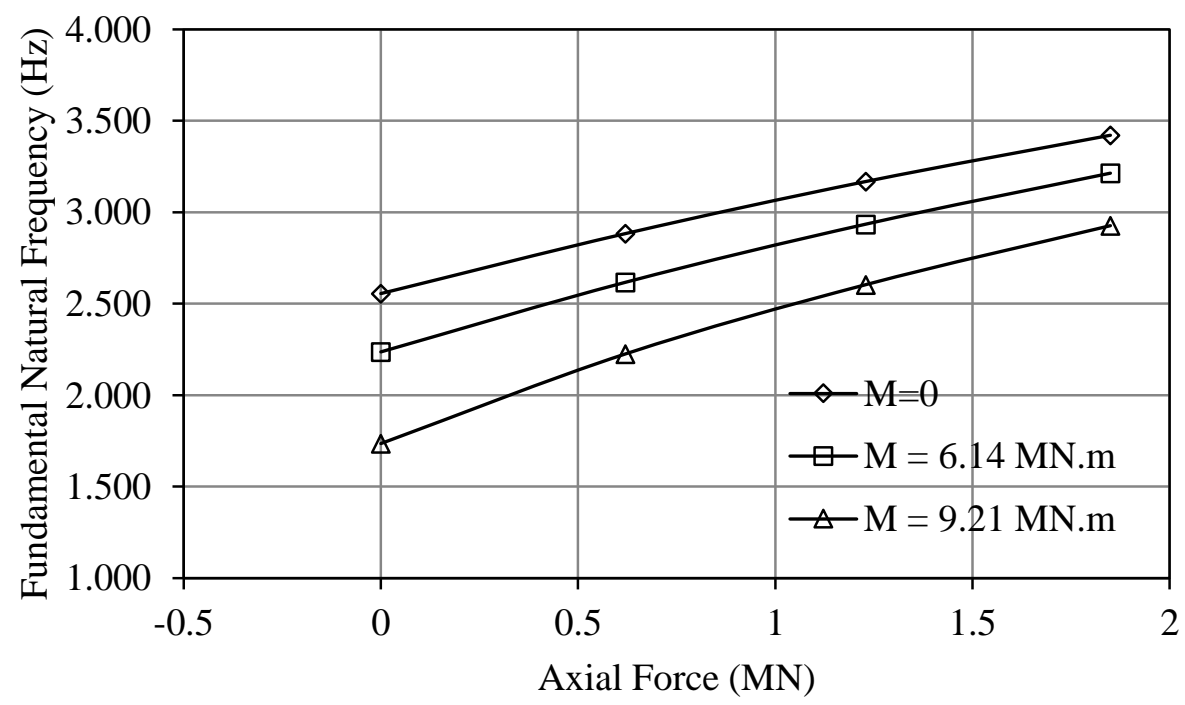

Figure 14: Variation of natural frequencies when tensile force and end-moment is applied for cantilevered $(C-F)$ boundary condition 


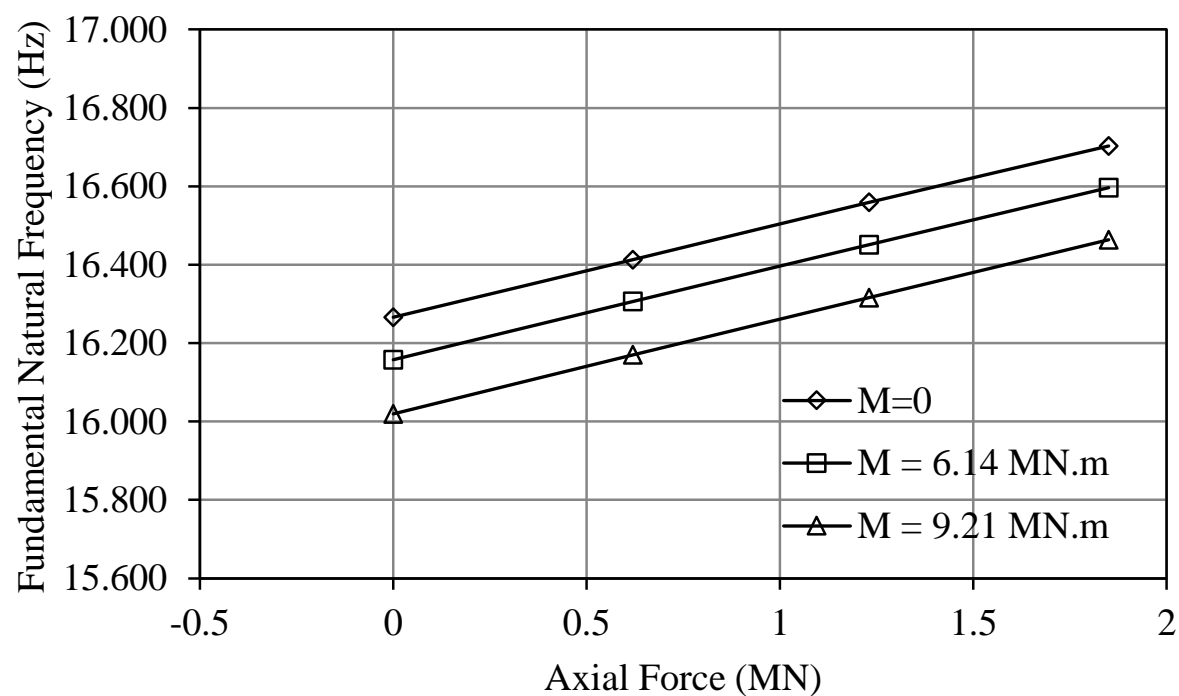

Figure 15: Variation of natural frequencies when tensile force and end-moment is applied for clamped - clamped $(C-C)$ boundary condition

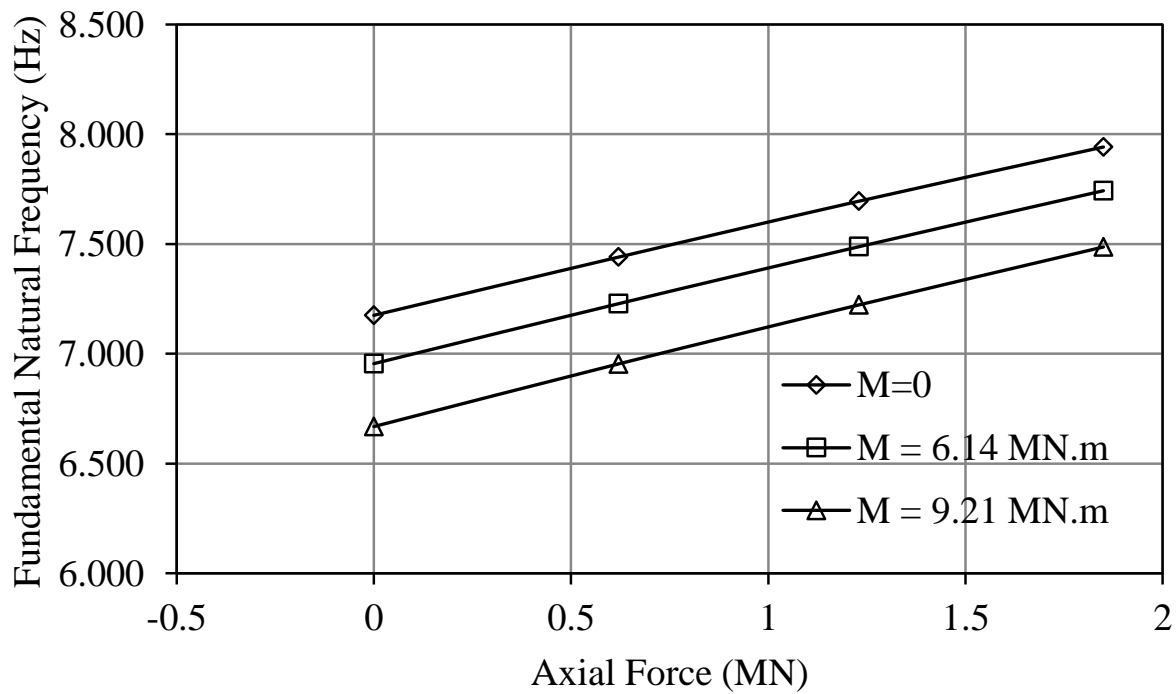

Figure 16: Variation of natural frequencies when tensile force and end-moment is applied for pinned - pinned $(P-P)$ boundary condition 


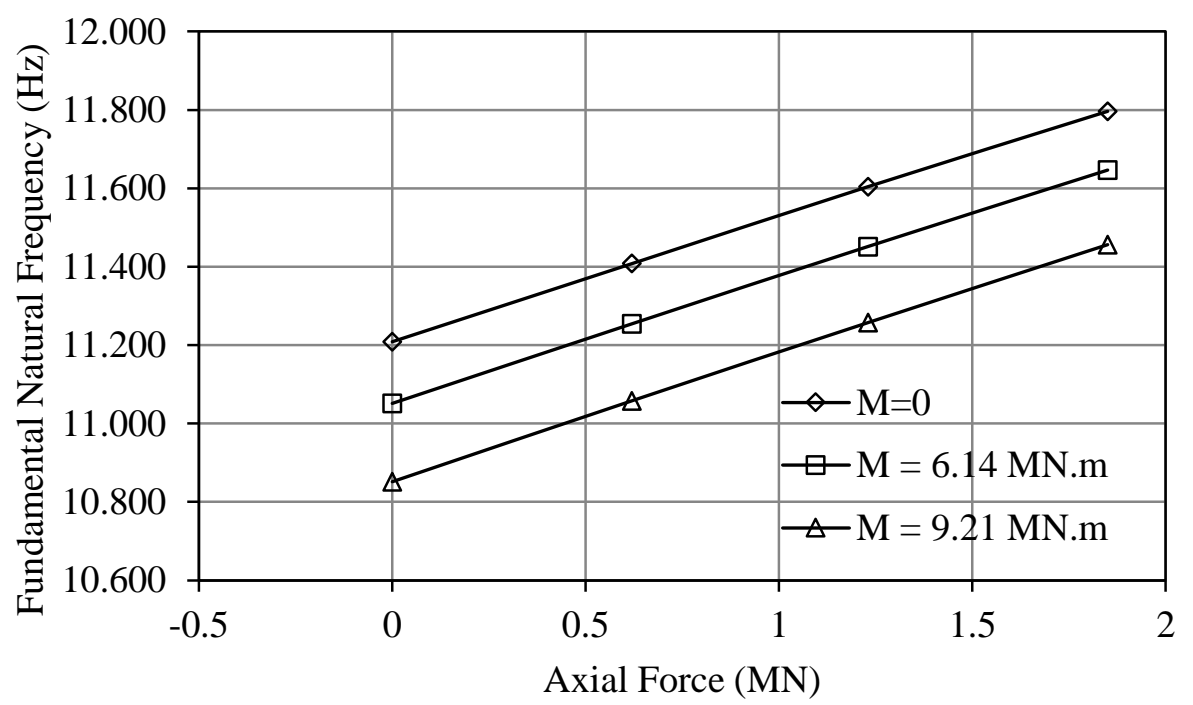

Figure 17: Variation of natural frequencies when tensile force and end-moment is applied for pinned - clamped $(P-C)$ boundary condition

Figure 18 below illustrates how the critical buckling end-moment varies with axial force. Figure 19 depicts the fluctuation of the critical buckling compressive force with changing end-moment.

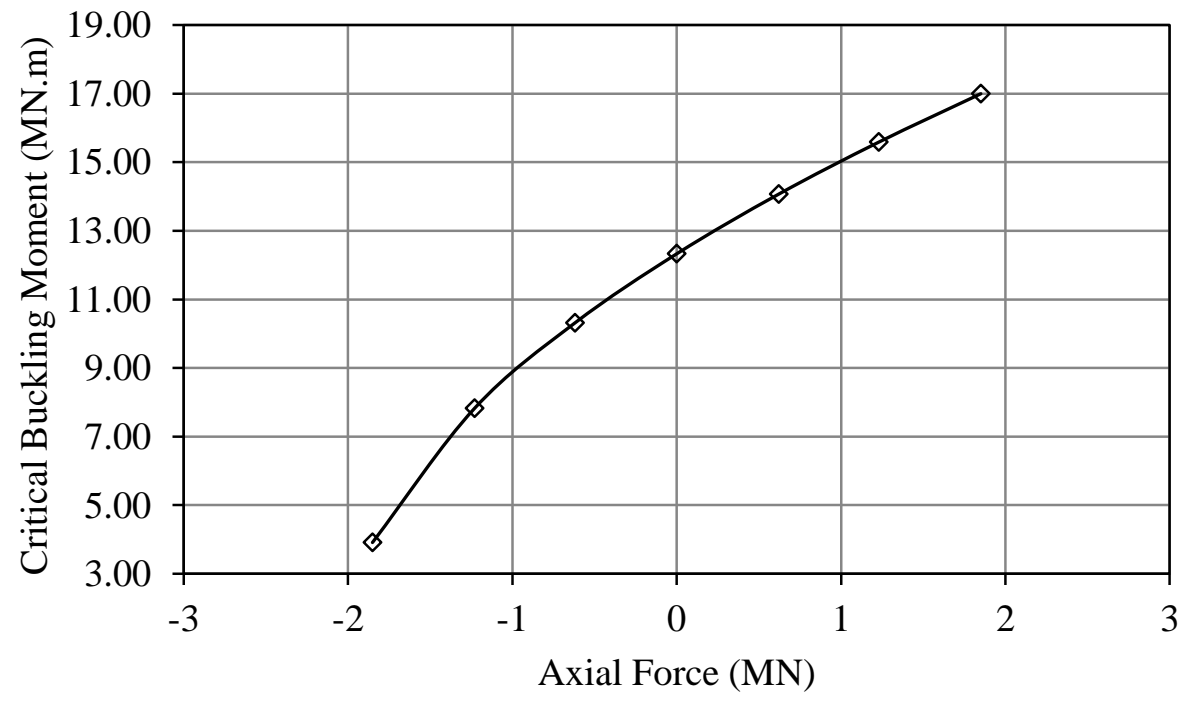

Figure 18: Variation of critical buckling end-moment with axial force for cantilevered $(C-F)$ boundary condition 


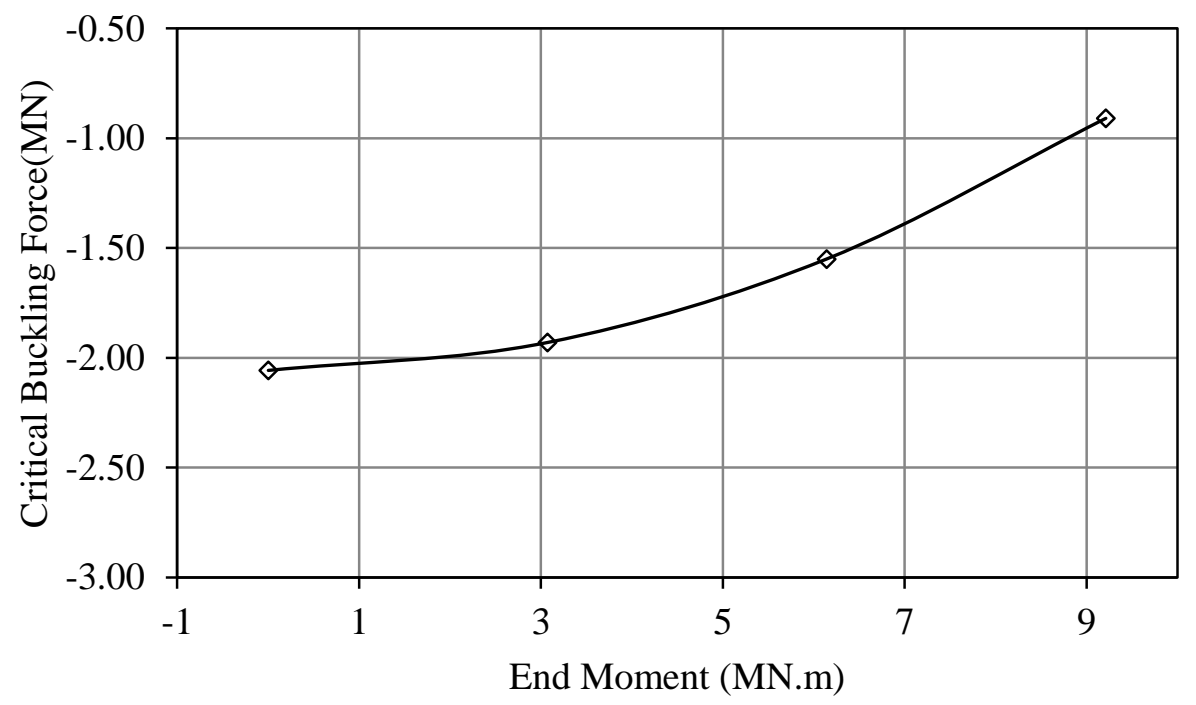

Figure 19: Variation of critical buckling compressive force with end-moment for cantilevered $(C-F)$ boundary condition

Figure 20 and Figure 21 show the bending and torsional components of mode shapes of a cantilever beam when a force of $1.85 \mathrm{MN}$ and moment of $9.21 \mathrm{MN} . \mathrm{m}$ is applied, respectively.

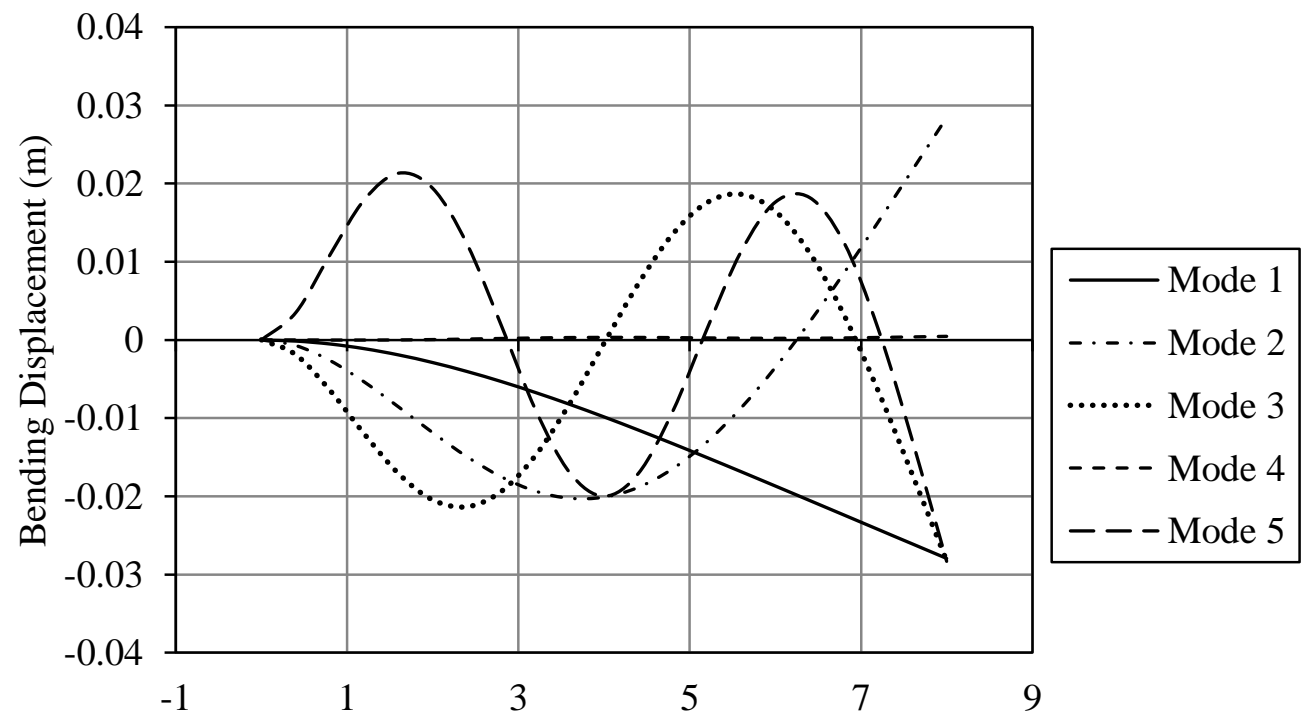

Distance from Clamped End (m)

Figure 20: Bending component of mode shapes of the system 


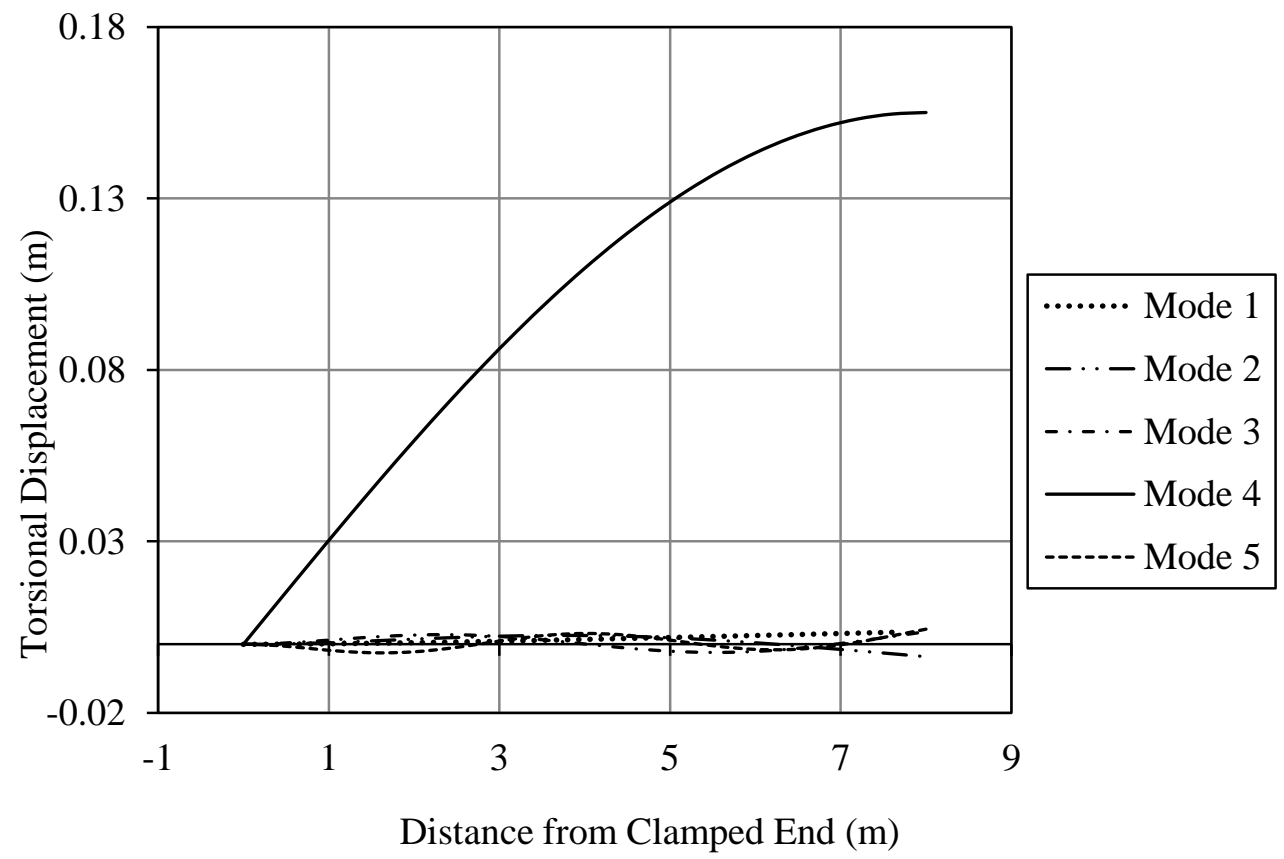

Figure 21: Torsional component of mode shapes of the system

The results obtained using the DFE method are in excellent agreement with the results of the conventional FEM for all types of loading and classical boundary conditions, as can be observed from Tables 9 through 12. Once again it is evident that the natural frequency of the system increases when the tensile axial force is applied. Any increment in the magnitude of the tensile force further increases the natural frequency of the system suggesting of a simultaneous increment in the stiffness of the beam. This is further confirmed in Figure 18 and Table 13 as it could be observed that a tensile force of $1.85 \mathrm{MN}$ causes the critical buckling moment to increases to 17 MN.m. Requiring a larger moment to cause buckling indicates a stiffening of the beam.

The DFE method further confirms that application of an end-moment reduces the stiffness of the beam. If the magnitude of the end-moment is increased, the value of the fundamental natural frequencies reduces proportionately. This observation is supported by the results included in 
Figure 19 and Table 14 which show that the higher the end-moment applied, the smaller the compressive force required to buckle the beam. If the beam buckles due to a small compressive force, this means that it has lost stiffness due to the moment. Tables 9 through 12 also include the effect of the end-moment and tensile force when they are acting together. If the tensile force is kept constant and the end-moment is increased, the natural frequencies of the beam reduce. The opposite happens, if the end-moment is unchanged while the tensile force is increased. All the above observations hold true for all classical boundary conditions considered in this study. Graphical representations of the results in Tables 9 through 12 are shown in Figures 14 through 17, respectively. It could also be observed that Figures 14 through 17 that were generated using the results of the DFE method are almost identical to Figures 4 through 7 produced using FEM results. Thus, the DFE results agree well with the findings from the FEM method.

Results from the DFE method also confirm that unlike tensile force, application of compressive force causes a reduction in the stiffness of the beam which is accompanied by a simultaneous reduction in the natural frequencies of the system. As seen from Figure 18 and Table 13, when no axial load is applied, the critical buckling moment of the beam is $12.33 \mathrm{MN}$. However, when a compressive force of $-1.85 \mathrm{MN}$ is acting, the critical buckling end-moment reduces to 3.91 MN.m, which is less than the critical moment when no forces are acting whatsoever. The results in Figure 19 and Table 14 further confirm this. Figure 19 and Table 14 show that if the compressive force is reduced to $-0.91 \mathrm{MN}$ a very large buckling moment of 9.21 MN.m is required to cause elastic instability of the beam, however, if the compressive force is increased to $-2.06 \mathrm{MN}$ the beam would buckle even without the presence of an end-moment. These results are true for all boundary conditions and almost identical to the findings from the conventional FE method. Thus, the 
findings of the DFE method agree with the findings in the literature that a compressive axial load reduces the fundamental frequencies as well as the stiffness of a beam.

Figure 20 illustrates that bending displacement is predominant until the fourth mode is reached. In Figure 20, the fourth mode shape shows very little bending displacement compared to the rest of the modes. However, in Figure 21 the fourth mode shows significant magnitudes of torsional displacement. Thus, the fourth mode shape is the first major torsional mode shape. The conclusion that the $1^{\text {st }}, 2^{\text {nd }} 3^{\text {rd }}$ and $5^{\text {th }}$ modes are predominantly flexural is confirmed by the fact that these mode shapes show very little torsional displacement in Figure 21. The mode shapes generated using the DFE method are very much identical to those produced by the FEM method seen in Figure 10 and Figure 11. Figures 31 through 35 in the Appendix B depict the coupled bending torsion mode shapes generated using ANSYS. Figure 34 from ANSYS, confirms that the fourth mode is the first predominantly torsional mode. 


\section{Concluding Remarks and Recommendations for Future Works}

The study confirms the findings from past literature that tensile forces increase the natural frequencies and stiffness of a beam while compressive forces and end-moments reduces the natural frequencies and stiffness of a beam for all classical boundary conditions. The results determined using the DFE method are in agreement with the results found from the FE method.

Most importantly, this study demonstrates that the DFE method could be extended to analyse systems exhibiting geometric couplings due to the presence of an end-moment. The Dynamic Finite Element (DFE) method is a superconvergent method that requires significantly less elements compared to the conventional FEM method and as such is very efficient. If the system is uncoupled, the DFE method is capable of producing exact results with just a single element as it utilizes the exact solutions to the uncoupled governing differential equations as dynamic basis functions of approximation space to develop the frequency dependent trigonometric shape functions. Even for a coupled system, the DFE method requires much less elements compared to the FE method.

Another advantage of the DFE method is that, unlike the conventional FE method, it allows the user to determine if a natural frequency of the system exists within a given range of frequencies. This is very important for aerospace applications where the range of operating frequencies of a certain component is already known, since the DFE method could then be used to determine if a natural frequency of the component occurs within the range of its operational frequencies. It would allow the designers to eliminate or limit the risk of resonance. Apart from the inherent advantage of allowing for a much coarser mesh, thus resulting in a largely simplified Eigenvalue problem due to the lesser number of elements compared to the conventional FEM method, the DFE method is also a powerful tool that could be used during the preliminary design stages to arrive at natural 
frequencies and mode shapes of a component with acceptable precision within a very short time. Especially during the early design stages for aerospace components that could be modelled as beams, such as helicopter, propeller and compressor blades or for structures such as planar or space frames that could be represented by assemblages of identical beams, the DFE beam model would be useful as the coupled natural frequencies of these components are necessary for aeroelastic calculations.

For future works, the effects of warping for torsion could be included to take in to account thinwalled beams and beams with open cross sections. Furthermore, the effects of shear could also be investigated. The DFE beam model presented here could be used as a base on to which more complex features such as geometric nonlinearity and variation of material properties are incorporated in the future. Although, in this study the frequency domain was swept using visual inspection to determine the system natural frequencies, an improvement could be made to the method by employing the Wittrick - Williams [16] root finding algorithm for determinant search, thus ensuring that no natural frequencies are missed. Furthermore, the DFE method could be used to determine the vibration and stability of Timoshenko beams and layered (hybrid) beams.

Finally, in the future the DFE method could also be extended to carry out vibrational analyses of two dimensional elements such as plates. As the Dynamic Finite Element method described in this study is not only limited to beams, it could be extended to plate structures which are more commonly used to model fuselage and wing skins. As the geometries and material properties of these structures are very complex, future studies could also focus on developing robust DFE formulations of plate structures that could be conveniently used to model the skins of fuselages and wings during preliminary design stages. 


\section{Appendix A: Shape Functions}

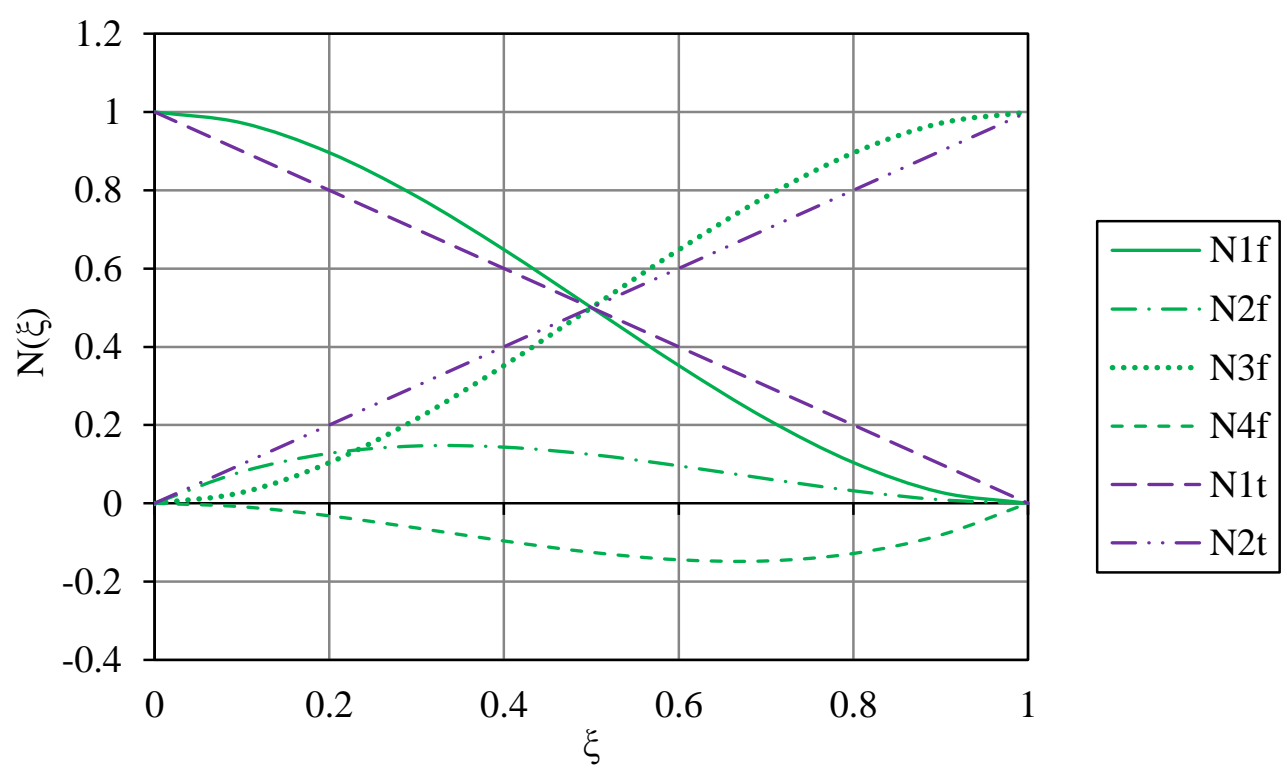

Figure 22: Cubic Hermite shape functions for bending and linear shape functions for torsion used in conventional FEM

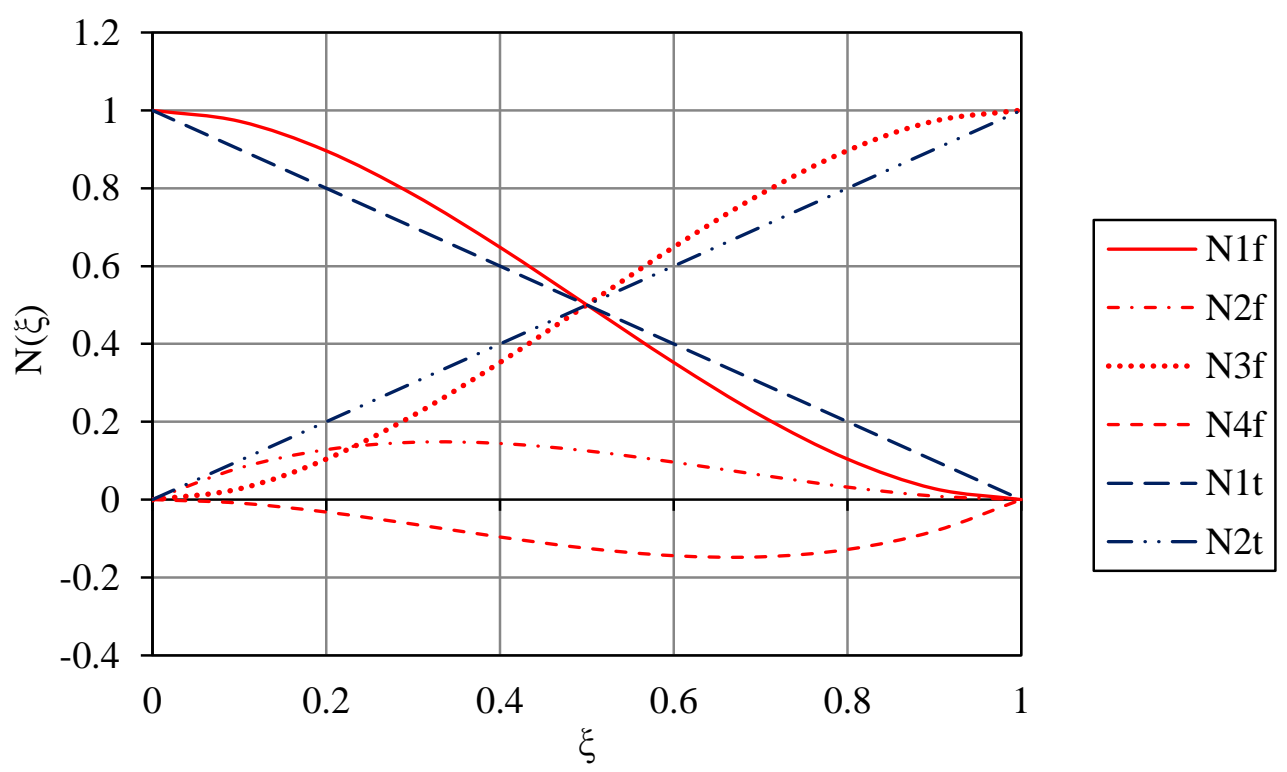

Figure 23: Frequency dependent trigonometric bending and torsion shape functions presented in [1] and [23] used in the DFE method 


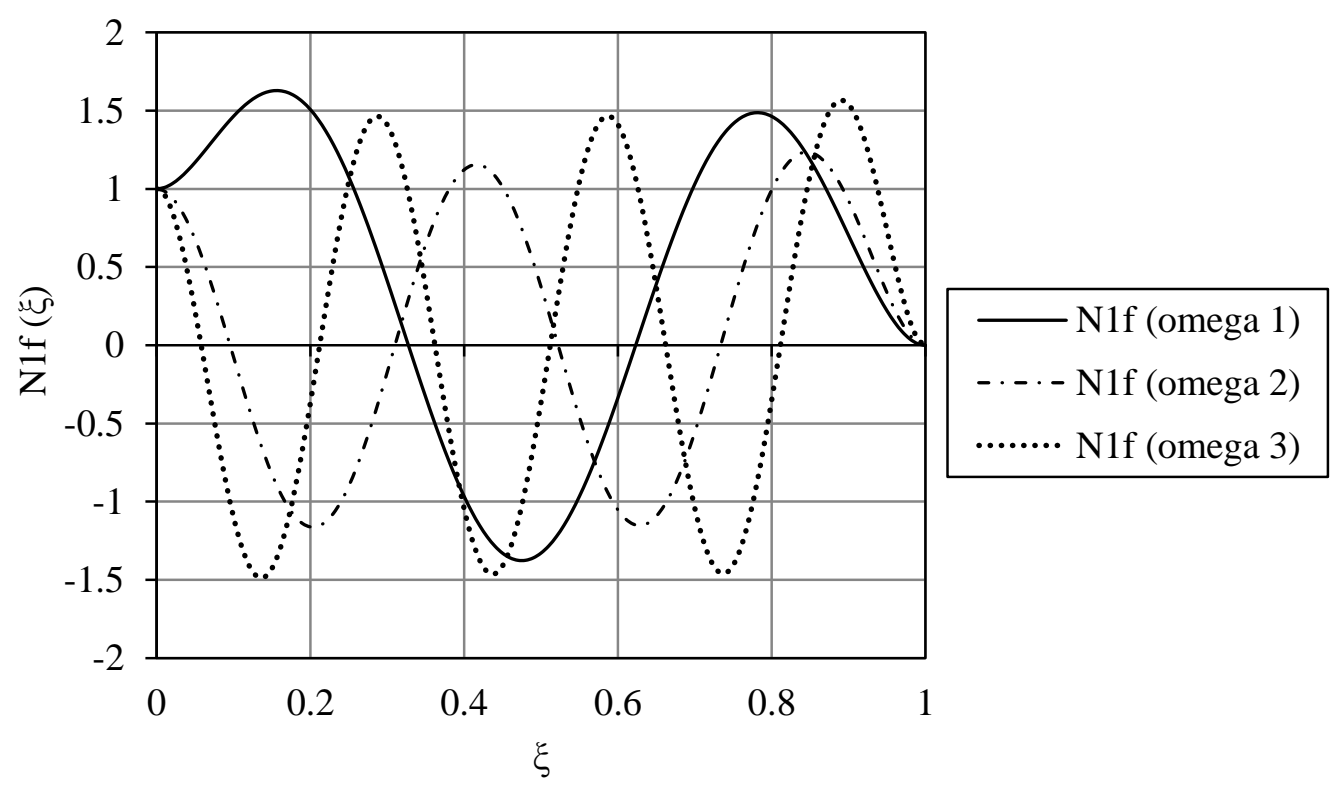

Figure 24: Dependency on the frequency of the first bending shape function [1,23] used in the DFE method

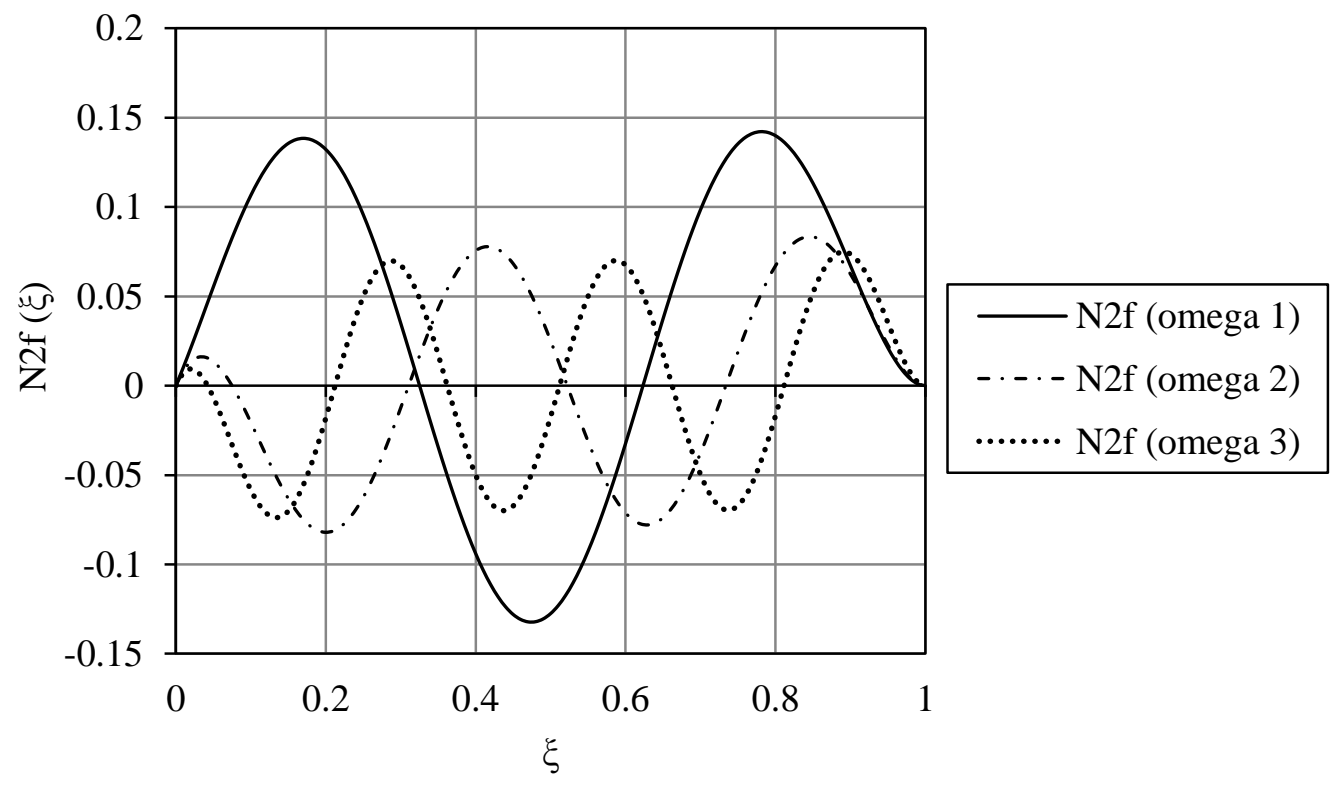

Figure 25: Dependency on the frequency of the second bending shape function [1,23] used in the DFE method 


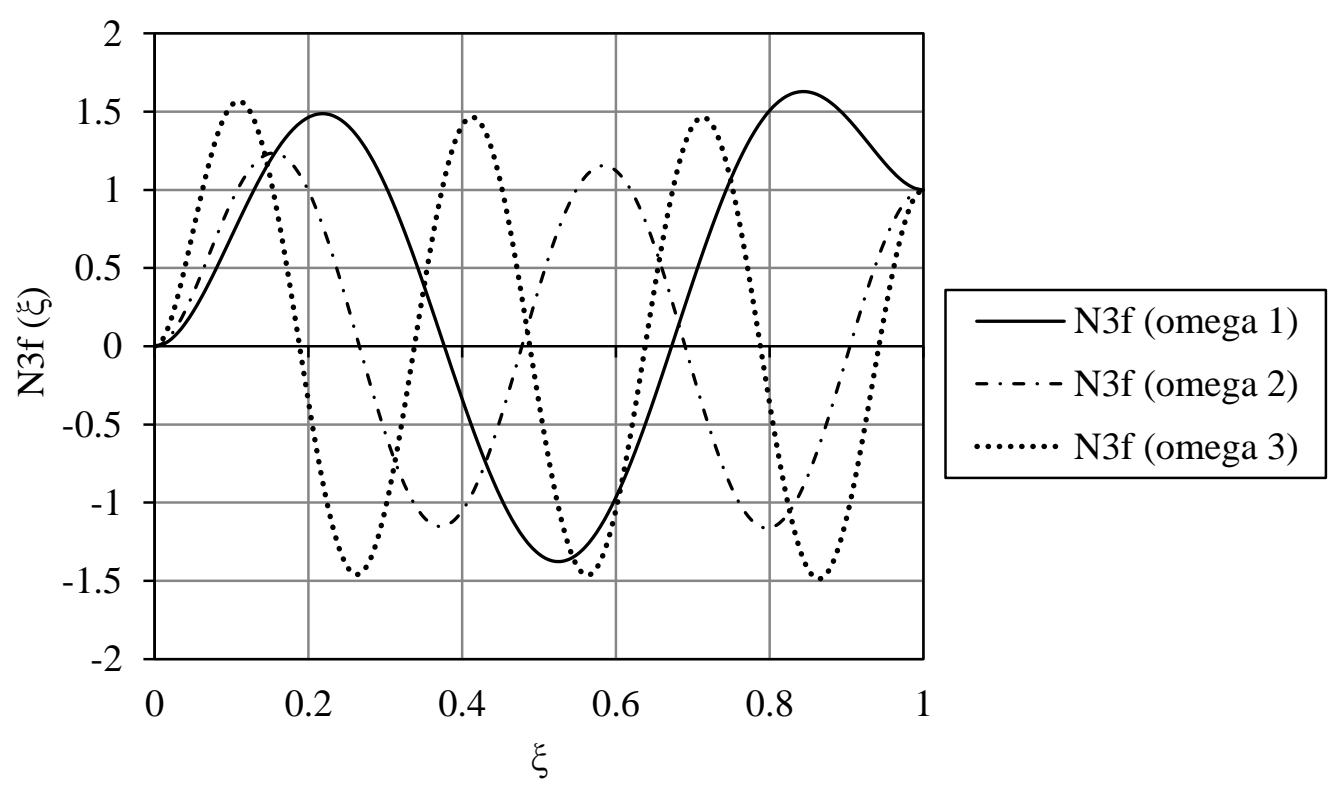

Figure 26: Dependency on the frequency of the third bending shape function [1,23] used in the DFE method

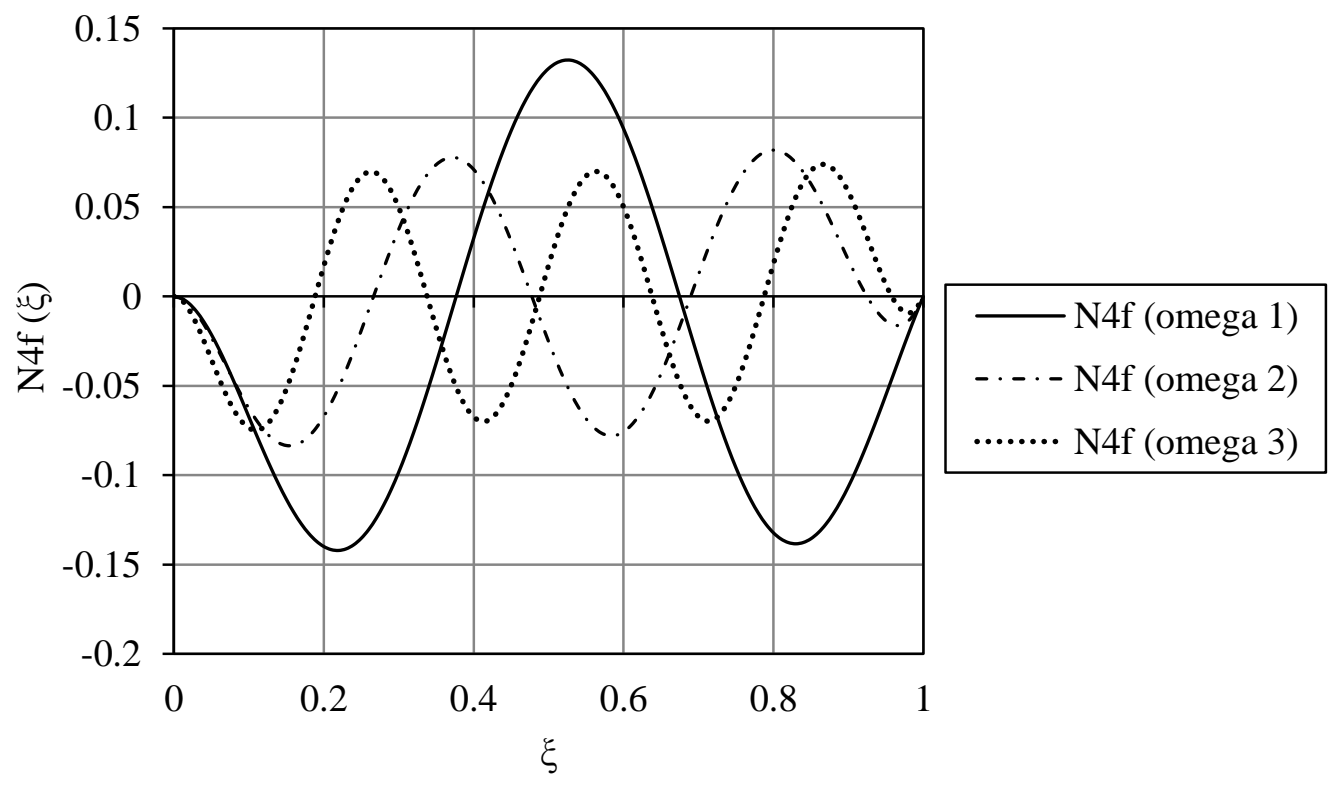

Figure 27: Dependency on the frequency of the fourth bending shape function [1,23] used in the DFE method 


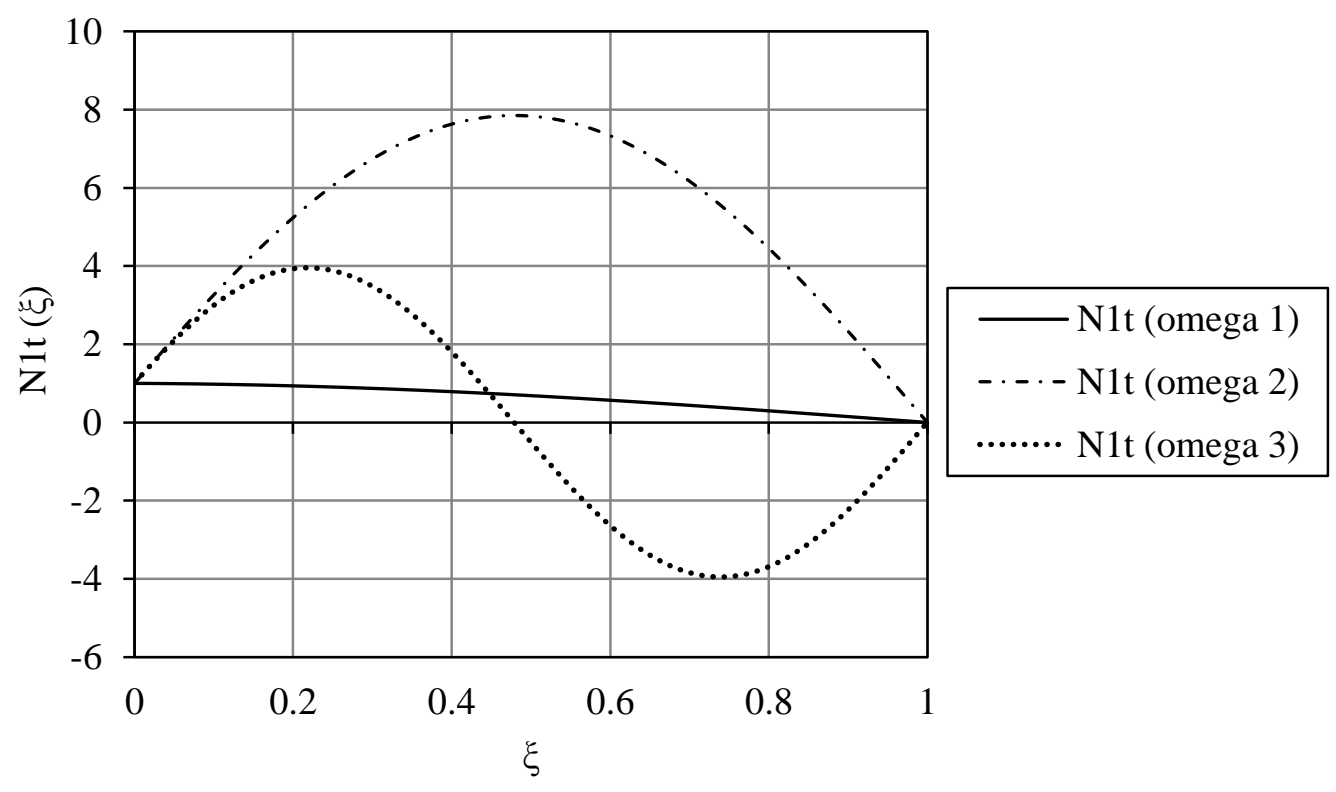

Figure 28: Dependency on the frequency of the first torsional shape function [1,23] used in the DFE method

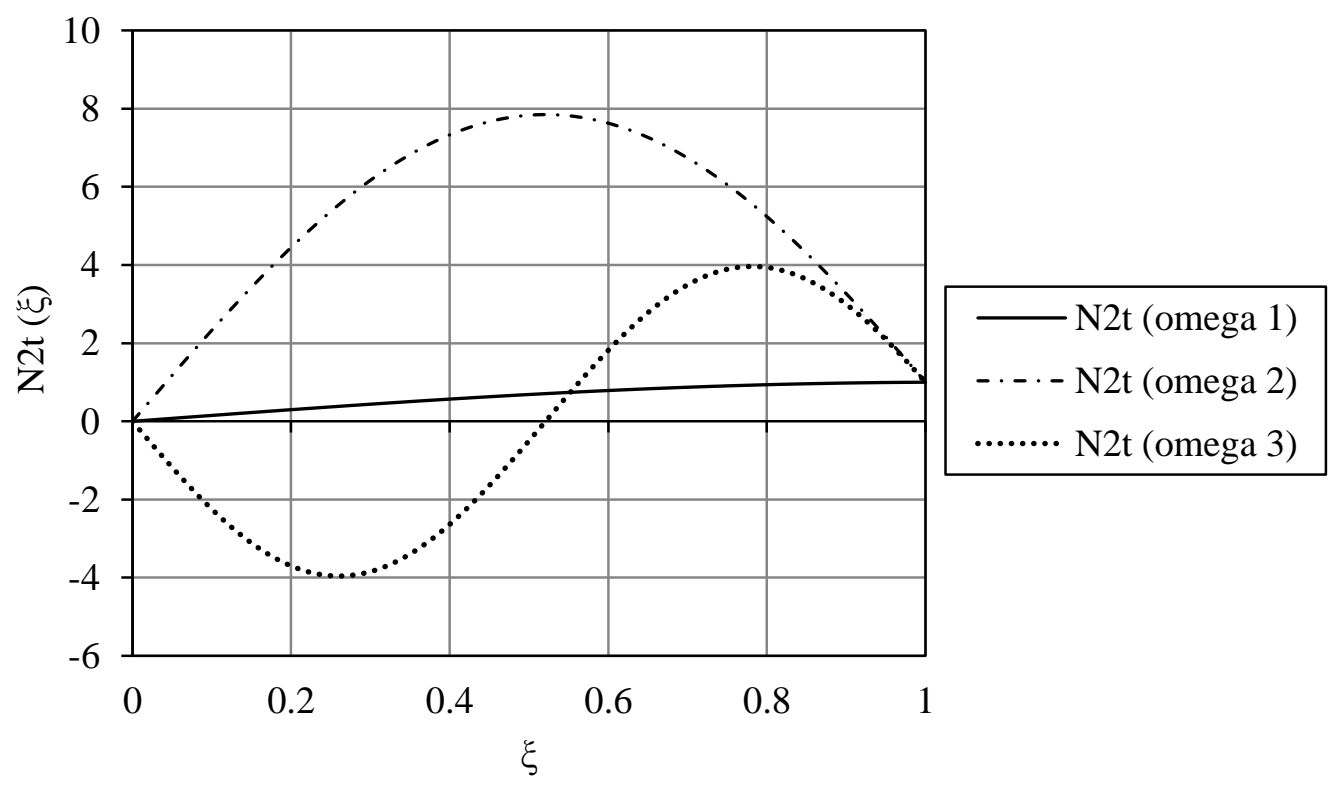

Figure 29: Dependency on the frequency of the second torsional shape function [1, 23] used in the DFE method 


\section{Appendix B: ANSYS Model}

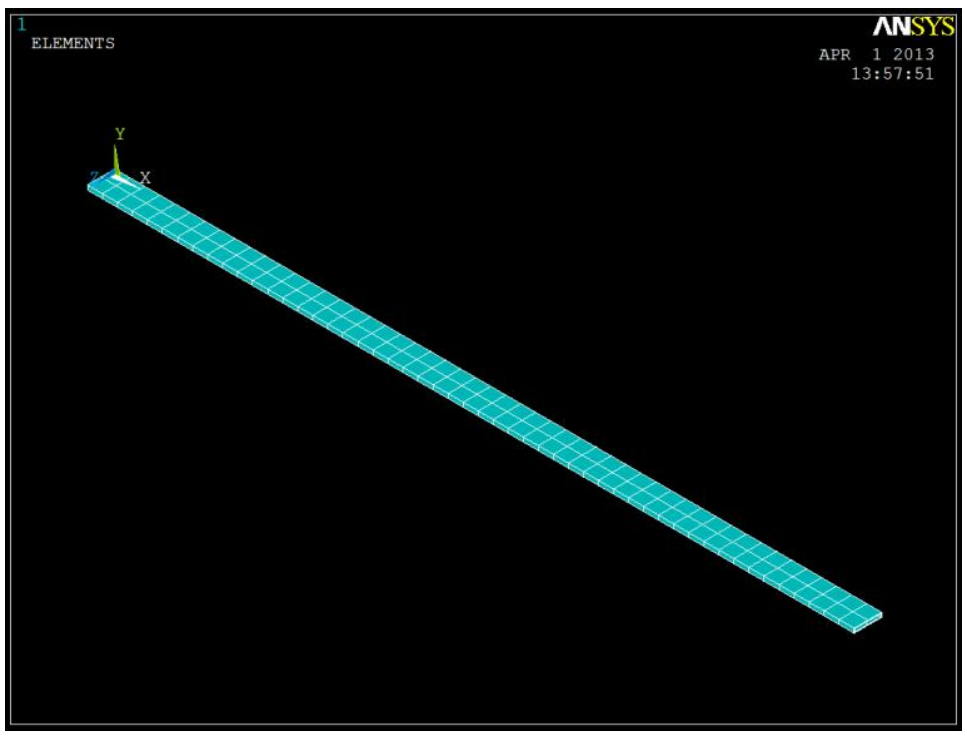

Figure 30: Beam meshed in ANSYS using SOLID 187 elements

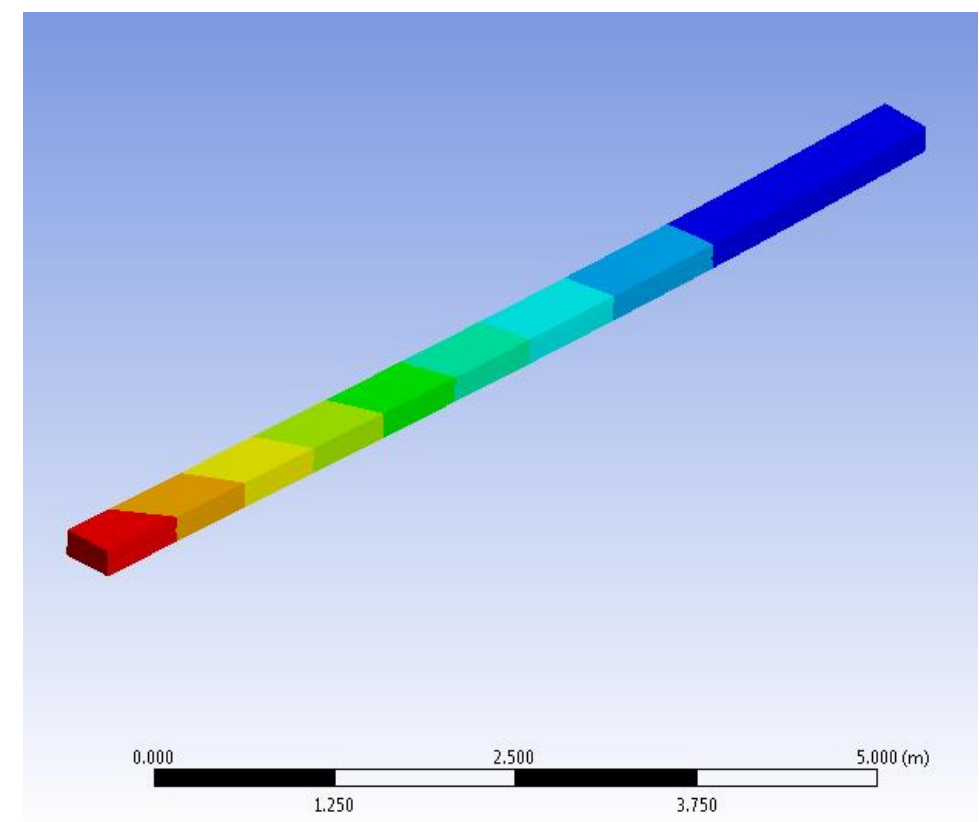

Figure 31: First coupled bending - torsion mode shape from ANSYS 


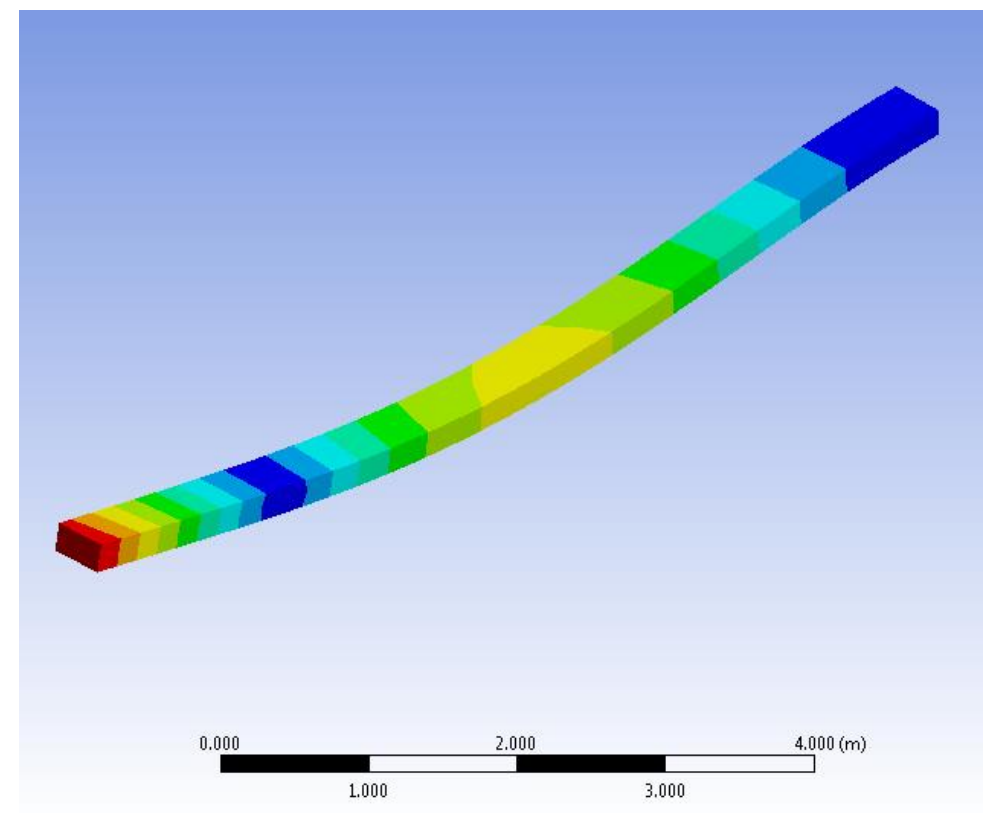

Figure 32: Second coupled bending - torsion mode shape from ANSYS

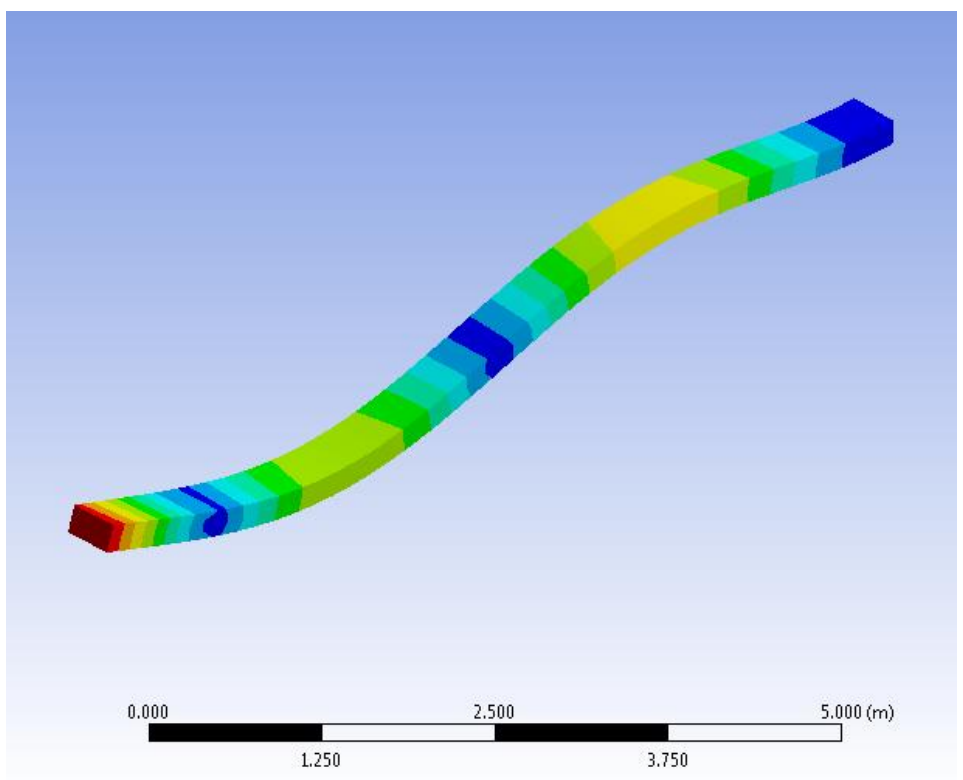

Figure 33: Third coupled bending - torsion mode shape from ANSYS 


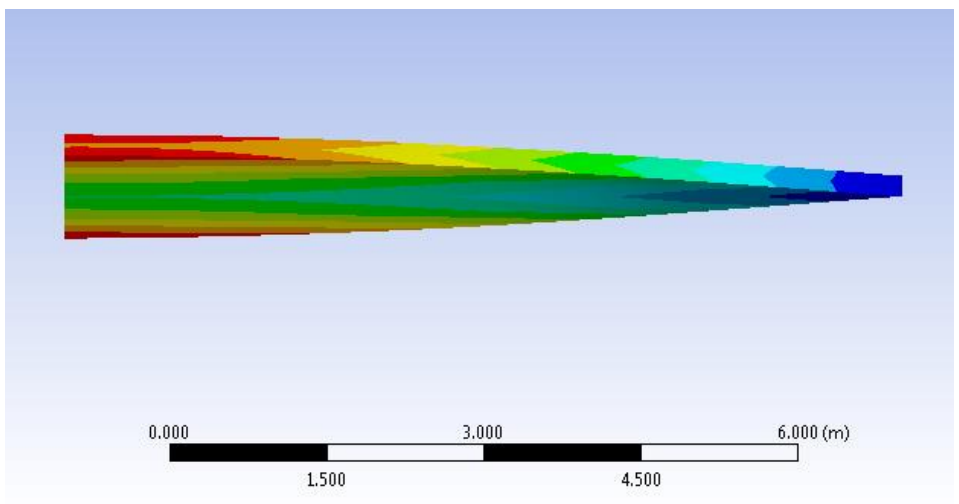

Figure 34: Fourth coupled bending - torsion mode shape from ANSYS

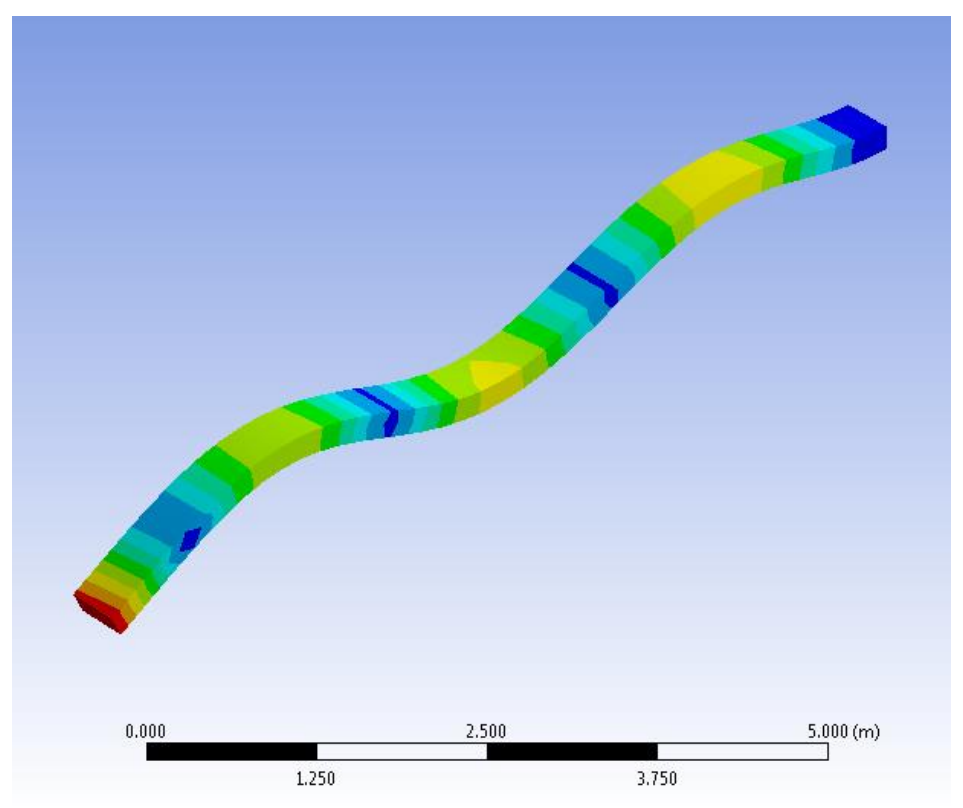

Figure 35: Fifth coupled bending - torsion mode shape from ANSYS 


\section{References}

1. Hashemi, S., Richard, J.M., (2000). A DFE method for free vibrations of bending - torsion coupled beams. Aerospace Science and Technology. 4: $41-55$.

2. Hashemi, S.M. and Roach, A., (2010). A Dynamic Finite Element for the Free Vibration Analysis of Extension-Torsion Coupled Composite Beams. Mathematics in Engineering, Science and Aerospace (MESA), The Transdisciplinary International Journal. 1(3): 221 239.

3. Hashemi, S.M., and Adique, E.J., (2010). A Quasi - Exact Dynamic Finite Element for Free Vibration Analysis of Sandwich Beams. Applied Composite Materials. 17: 259 - 269.

4. Borneman, S., Hashemi, S. (2004). Dynamic Finite Element (DFE) Formulation for the Free Vibration Analysis of Laminated Composite Wing - Beams. Journal of Sound and Vibration.

5. Dokumaci, E., (1987). An exact solution for coupled bending and torsion vibrations of uniform beams having single cross sectional symmetry. Journal of Sound and Vibration. 119: $443-449$.

6. Mei, C. (1970). Coupled vibrations of thin-walled beams of open section using the finite element method. International Journal of Mechanical Science. 12:883 -91.

7. Wekezer, J.W., (1987).Free vibrations of thin-walled bars with open cross sections. Journal of Engineering Mechanics.113:1441 - 51.

8. Tanaka, M., Bercin, A.N., (1999). Free vibration solution for uniform beams of nonsymmetrical cross section using Mathematica. Computers and Structures. 71:1 - 8 .

9. Banerjee, J.R., Williams, F.W., (1994). Coupled bending - torsional dynamic stiffness matrix of an axially loaded Timoshenko beam element. International Journal of Solids and Structures. 31: $749-762$. 
10. Banerjee, J.R., Williams, F.W., (1995). Free vibration of composite beams - an exact method using symbolic computation. Journal of Aircraft. 32(3): 636 - 642 .

11. Banerjee, J.R., (2003). Free vibration of sandwich using the dynamic stiffness method. Computers \& Structures. 81: $1915-1922$.

12. Banerjee, J. R., Sobey, A.J., (2005). Dynamic stiffness formulation and free vibration analysis of a three - layered sandwich beam. International Journal of Solids and Structures. 42: $2181-2197$.

13. Banerjee, J. R., et al. (2007). Free vibration of a three - layered sandwich beam using the dynamic stiffness method and experiment. International Journal of Solids and Structures. 44: $7543-7563$

14. Banerjee, J.R., Su, H., and Jayatunga, C., (2008). A dynamic stiffness element for free vibration analysis of composite beams and its application to aircraft wings. Computers \&Structures. 86 (6): $573-579$.

15. Banerjee, J.R., Guo, S., Howson, W.P., (1996) Exact dynamic stiffness matrix of a bending -torsion coupled beam including warping. Computers and Structures. 59:613 - 21.

16. Wittrick, W.H., Williams, F.W., (1971). A general algorithm for computing natural frequencies of elastic structures. Quarterly Journal of Mechanics and Applied Mathematics.24: 263 - 284.

17. Bannerjee, J.R., Su, H., (2006). Free transverse and lateral vibration of beams with torsional coupling. Journal of Aerospace Engineering. 19: 1(13)

18. Borneman, S.R., Hashemi, S.M., and Alighanbari, H., (2008). Vibration Analysis of Doubly Coupled Cracked Composite Beams: An Exact Dynamic Stiffness Matrix. International Review of Aerospace Engineering (I.RE.AS.E.)1(3): 298 - 309. 
19. Friberg, P.O., (1985) Beam element matrices derived from Vlasov's theory of open thin walled elastic beams. International Journal for Numerical Methods in Engineering. $21: 1205-28$.

20. Leung, A.Y.T., (1991) Natural shape functions of a compressed Vlasov element. Thinwalled Structures.11:431 - 8.

21. Hallauer, W.L., Liu, R.Y.L., (1982). Beam bending - torsion dynamic stiffness method for calculation of exact vibration modes. Journal of Sound and Vibration. 85:105-13.

22. Timoshenko, S., (1964). Vibration problems in engineering. New York: Van Nostrand. See pp $374-377$.

23. Hashemi, S.M., Richard, M.J., (2000).Free vibrational analysis of axially loaded bending - torsion coupled beams: a dynamic finite element. Computers and Structures.77:711 - 24.

24. Banerjee, J.R., Williams, F.W., (1994). Coupled bending - torsional dynamic stiffness matrix of an axially loaded Timoshenko beam element. International Journal of Solids and Structures. 31: $749-762$.

25. Banerjee, J.R., Fisher, S.A., (1992). Coupled bending - torsional dynamic stiffness matrix for axially loaded beam elements. International Journal for Numerical Methods in Engineering. 33:739-51.

26. Banerjee, J.R., (1989). Coupled bending-torsional dynamic stiffness matrix for beam elements. International Journal for Numerical Methods in Engineering.28:1283 - 9.

27. Jun, L., et al (2004). Coupled bending and torsional vibration of axially loaded BernoulliEuler beams including warping effects. Applied Acoustics. 65: 153 - 170. 
28. Murthy, M., Neogy, J., (1969). Determination of fundamental natural frequencies of axially loaded columns and frames. Journal of the Institution of Engineers (India) Civil Engineering Division. 49: 203 - 212.

29. Gellert, M., Gluck, J., (1972). The influence of axial load on eigenfrequencies of a vibrating lateral restraint cantilever. International Journal of Mechanical Sciences 14: 723 - 728.

30. Bokaian, A., (1988). Natural frequencies of beams under compressive axial load. Journal of Sound and Vibrafion. 126 (1), $49-65$.

31. Bokaian, A., (1990). Natural frequencies of beams under tensile axial load. Journal of Sound and Vibration. 142 (3), $481-498$.

32. Shaker, F.J., (1975). Effect of axial load on mode shapes and frequencies of beams. NASA Technical Note. TN D - 8109.

33. Atsuta, T., Chen, W.F., (1977). Theory of beam columns - space behaviour and design volume New York: McGraw - Hill Book Co.

34. Joshi, A., Suryanarayan, S., (1984). Coupled flexural - torsional vibration of beams in the presence of static axial loads and end-moments. Journal of Sound and Vibration. 92: 583 -589 .

35. Joshi, A., Suryanarayan, S., (1989). A unified solution for the various boundary conditions for the coupled flexural - torsional vibration of beams subjected to axial loads and endmoments. Journal of Sound and Vibration.129: 313 - 326.

36. Joshi, A., Suryanarayan, S., (1984). A unified solution for various boundary conditions for the coupled flexural - torsional instability of closed thin - walled beam - columns. International Journal of Solid Structures. 20: 167 - 178. 
37. Joshi, A., Suryanarayan, S., (1991). Iterative method for coupled flexural - torsional vibration of initially stressed beams. Journal of Sound and Vibration. 146(1): 81 - 92.

38. Pavlovic, R., Kozic, P., (2003). Almost sure stability of the thin - walled beam subjected to end-moments. Theoretical Applied Mechanics. 30: 193 - 207.

39. Pavlovic, R., et al. (2007). Dynamic stability of a thin - walled beam subjected to axial loads and end-moments. Journal of Sound and Vibration. 301:690 - 700.

40. Meirovitch, L., (1967). Analytical Methods in Vibrations. The MacMillan Co.

41. Hashemi, S., Pereira, D., (2007). A Meshless Dynamic Finite Element for Beam Vibrations including Rotary Inertia. Proceedings of the $7^{\text {th }}$ International Conference on Computational Structures Technology. Stirlingshire, UK: Civil - Comp Press. Paper 127. 